\title{
Strong magnetic fields in lattice QCD
}

P.V.Buividovich, M.N.Chernodub,T.K. Kalaydzhyan, D.E. Kharzeev, E.V.Luschevskaya, M.I. Polikarpov

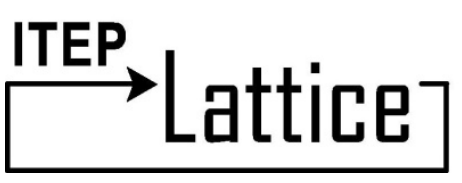

arXiv:1003.2180, arXiv:0910.4682, arXiv:0909.2350, arXiv:0909.1808, arXiv:0907.0494, arXiv:0906.0488, arXiv:0812.1740
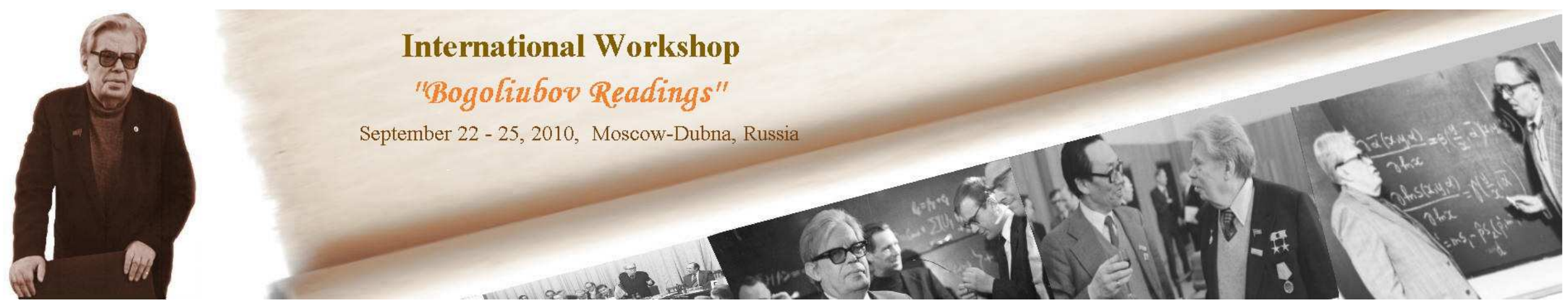


\section{Lattice simulations with magnetic fields}

1. Chiral Magnetic Effect

1.1 CME on the lattice

1.2 Vacuum conductivity induced by magnetic field

1.3 Quark mass dependence of CME

1.4 Dilepton emission rate

2. Other effects induced by magnetic field

2.1 Chiral symmetry breaking

2.2 Magnetization of the vacuum

2.3 Electric dipole moment of quark along the direction of the magnetic field
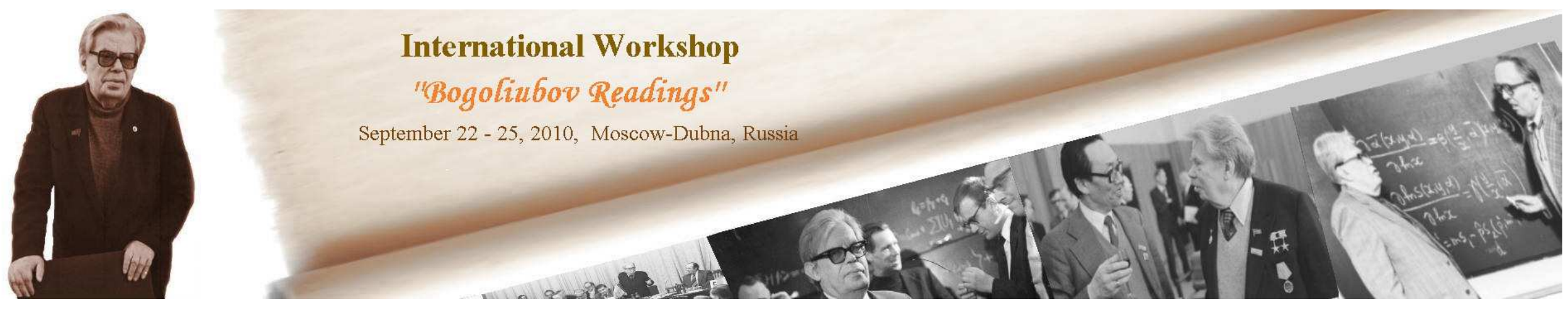
Main picture by McLerran,Kharzeev,Fukushima

\section{Magnetic fields in non-central collisions}

[Fukushima, Kharzeev, Warringa, McLerran '07-'o8]

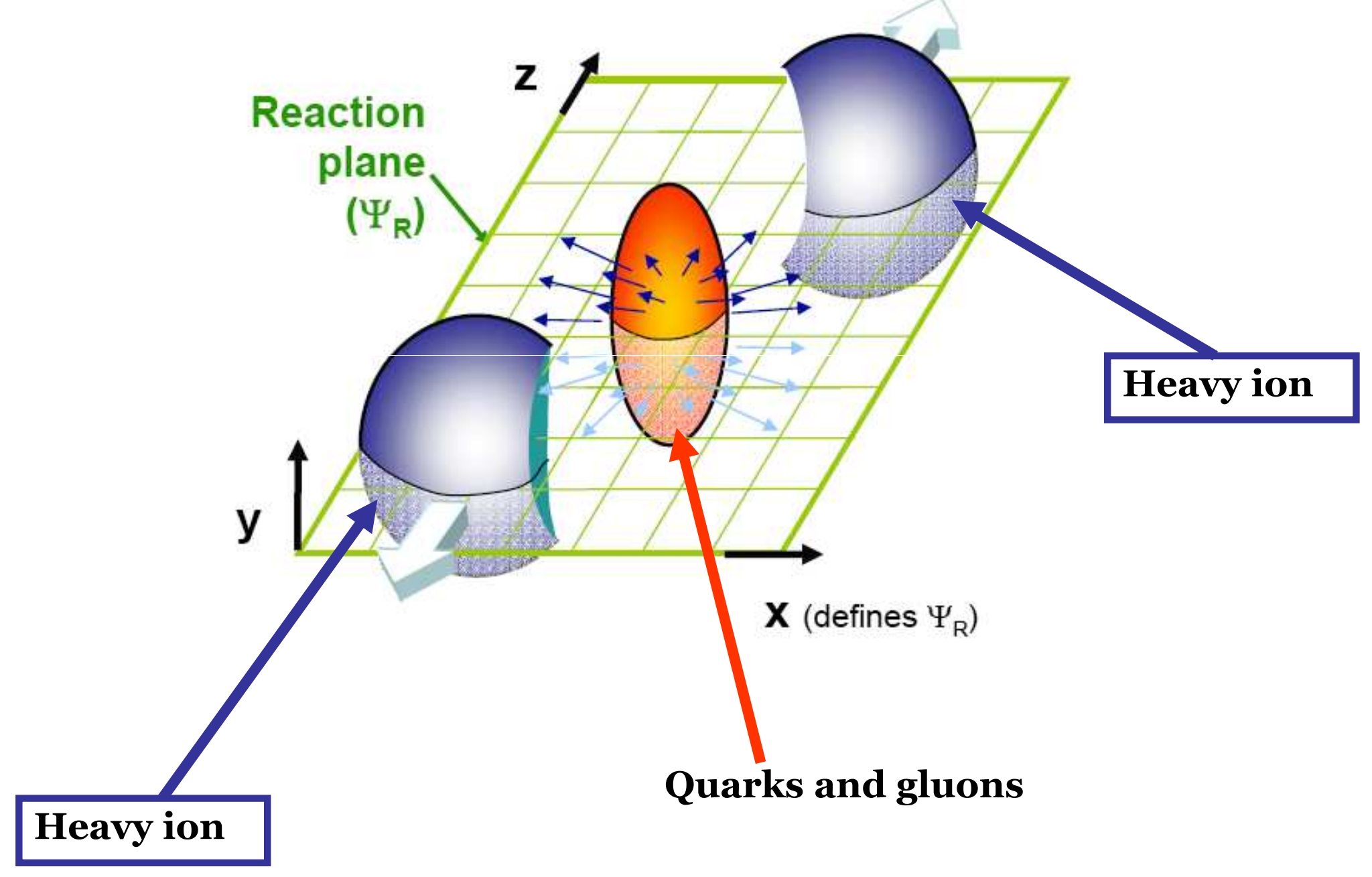


Main picture by McLerran,Kharzeev,Fukushima

\section{Magnetic fields in non-central collisions}

[Fukushima, Kharzeev, Warringa, McLerran '07-'o8]

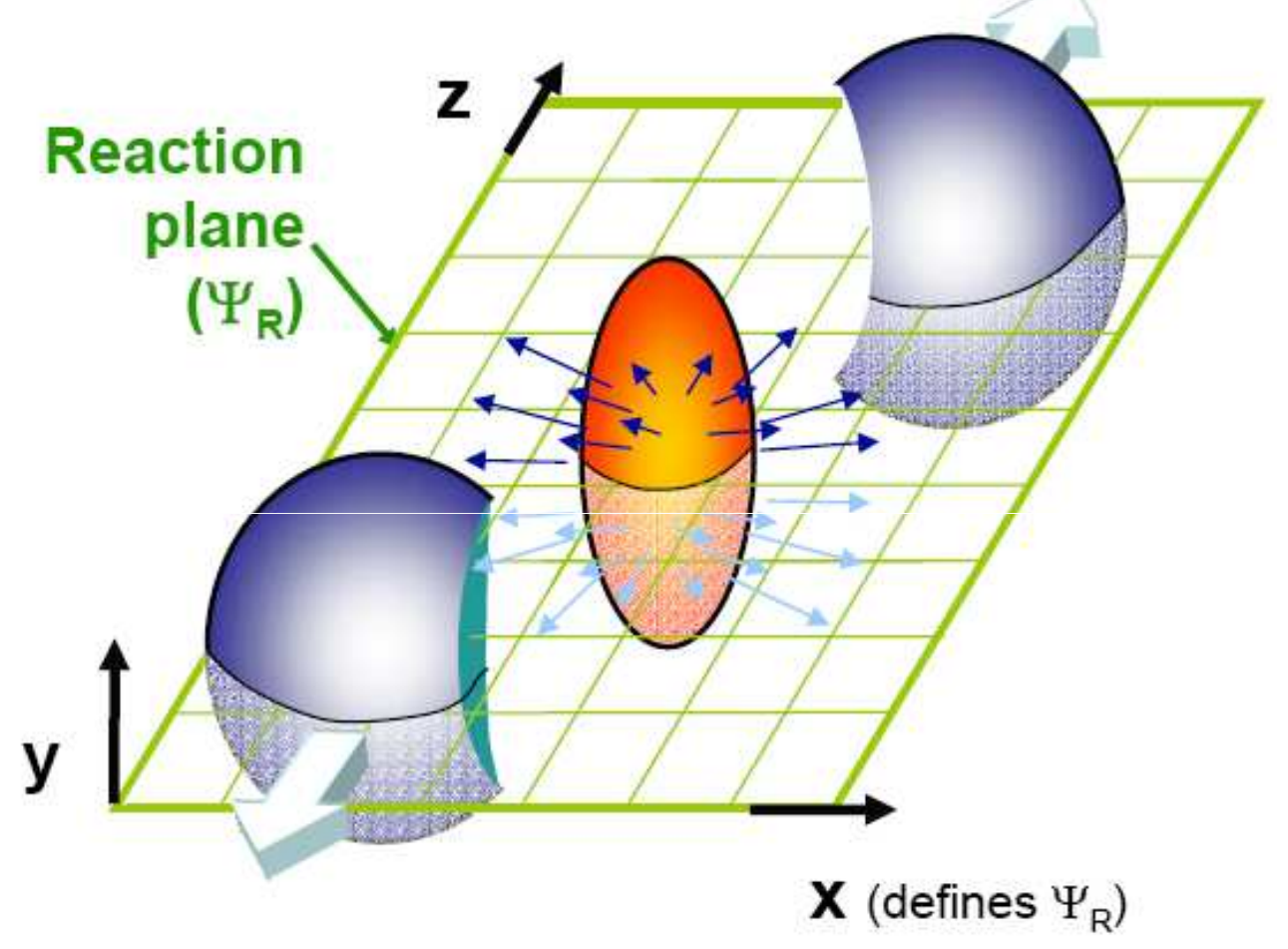

[1] K. Fukushima, D. E. Kharzeev, and H. J. Warringa, Phys. Rev. D 78, o74033 (20o8), URL http://arxiv.org/abs/o808.3382.

[2] D. Kharzeev, R. D. Pisarski, and M. H. G.Tytgat, Phys. Rev. Lett. 81, 512 (1998), URL http://arxiv.org/abs/hep-ph/9804221.

[3] D. Kharzeev, Phys. Lett. B 633, 260 (2006), URL http://arxiv.org/abs/hep-ph/o406125. [4] D. E. Kharzeev, L. D. McLerran, and H. J. Warringa, Nucl. Phys. A 803, 227 (2008),

URL http://arxiv.org/abs/o711.0950. 


\section{Magnetic fields in non-central pllisions}

The medium is fil.eu by electrically charged particles

Large orbital momentum, perpendicular to the reaction plane

Large magnetic field along the direction of the orbital momentum 
McLerran,Kharzeev,Fukushima Magnetic fields in

"Instanton"

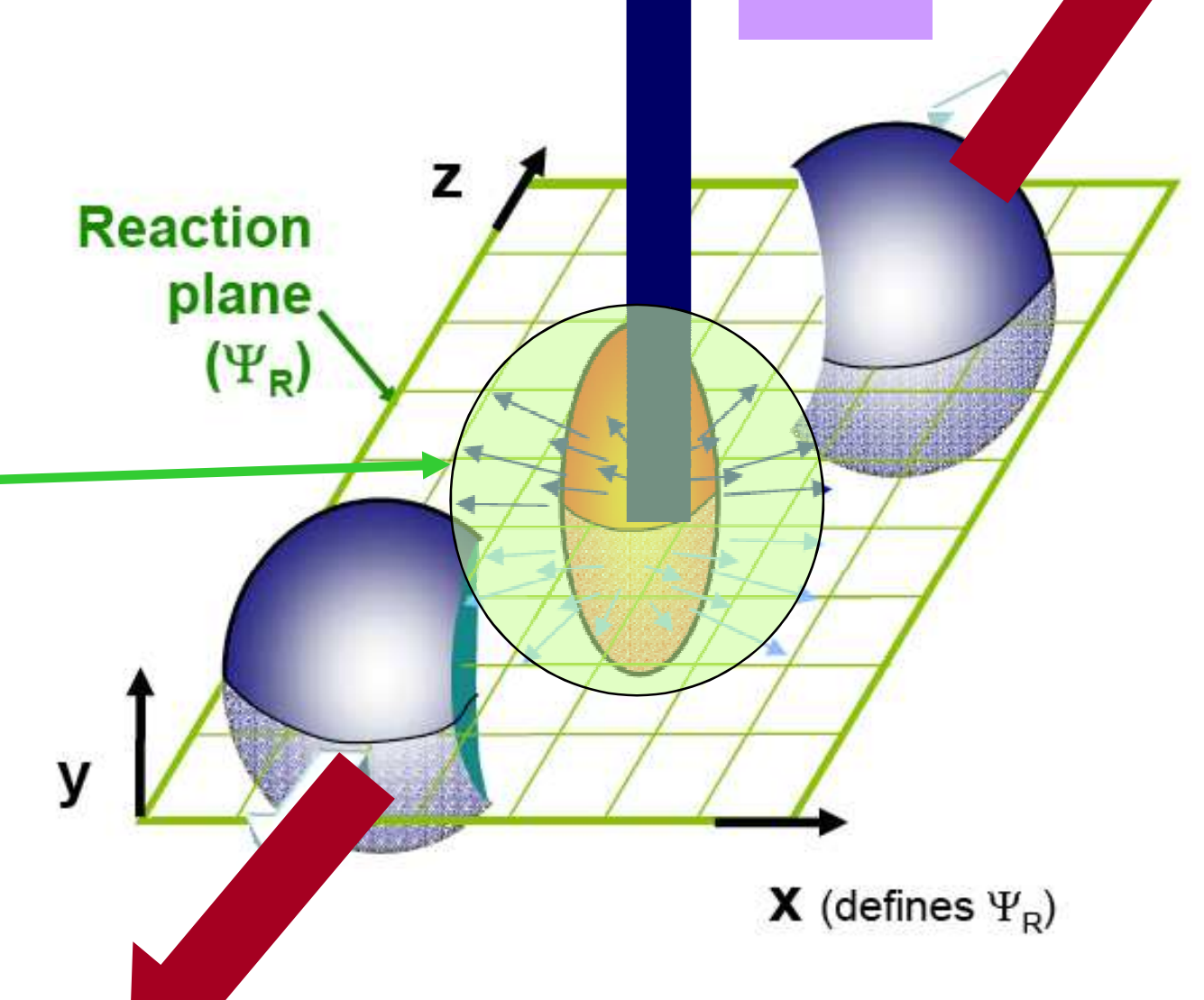

Two very big currents produce a very big magnetic field

The medium is fil.ea by electrically charged particles

Large orbital momentum, perpendicular to the reaction plane

Large magnetic field along the direction of the orbital momentum 


\section{In heavy ion collisions magnetic forces are of the order of strong interaction forces}

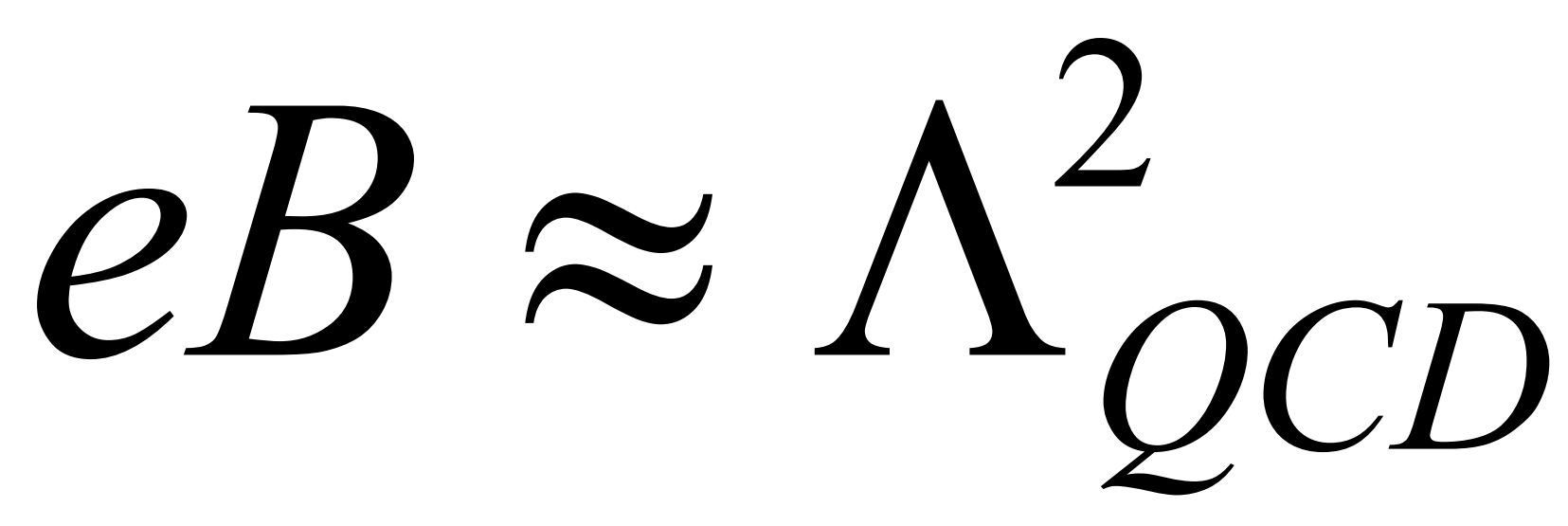




\section{Magnetic forces are of the order of strong interaction forces}

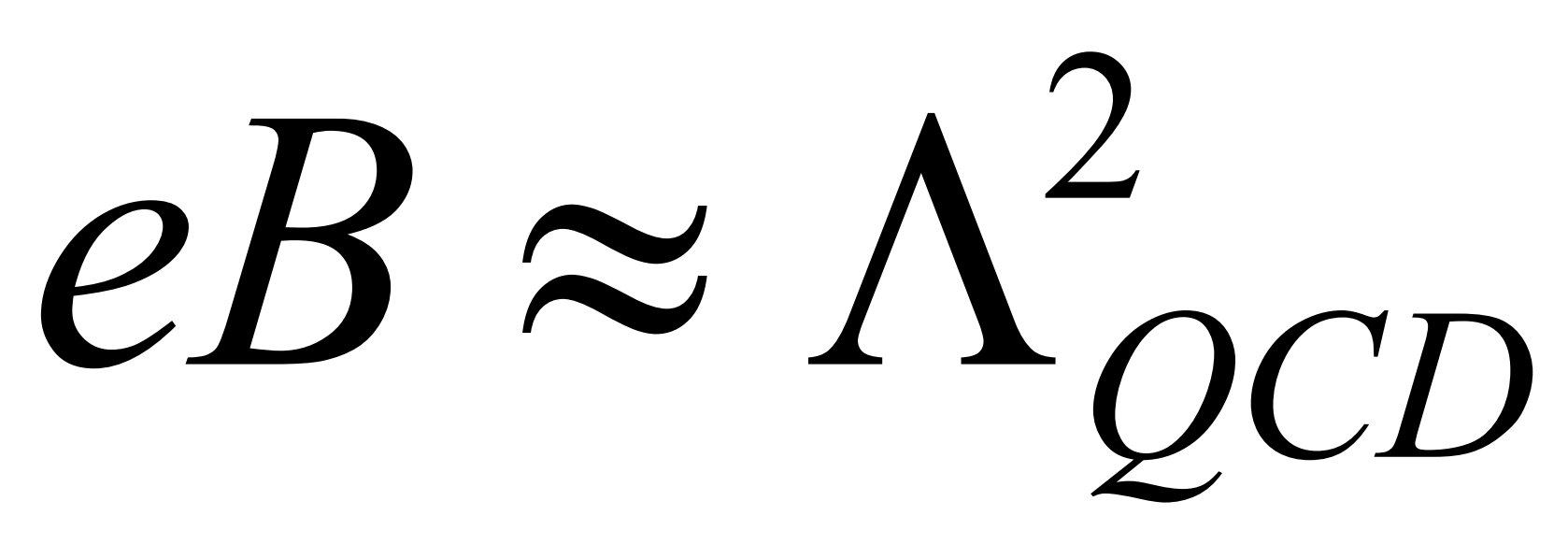

We expect the influence of magnetic field on strong interaction physics 


\section{Chiral Magnetic Effect by Fukushima, Kharzeev, Warringa, McLerran}

1. Massless quarks in external magnetic field.

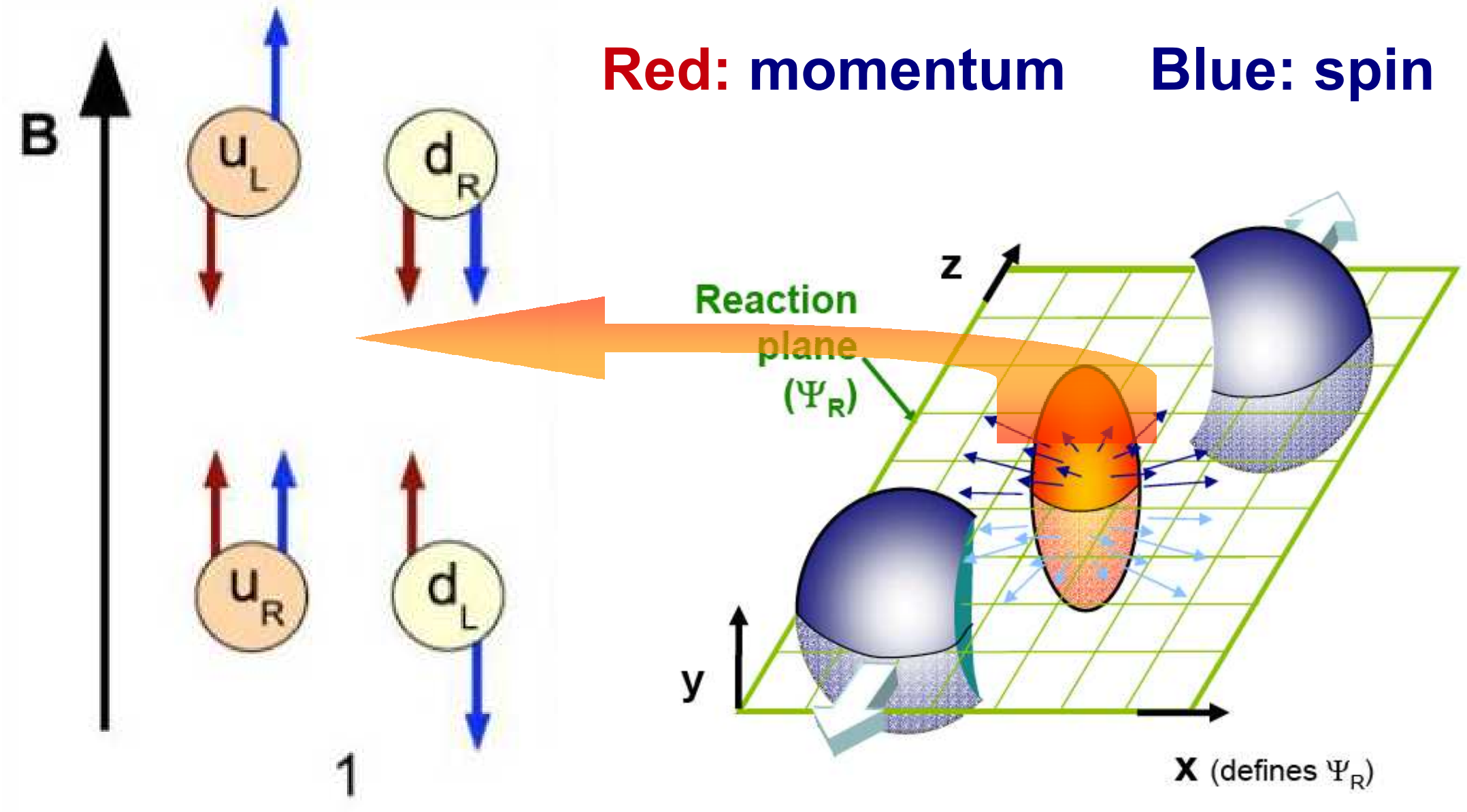




\section{Chiral Magnetic Effect by \\ Fukushima, Kharzeev, Warringa, McLerran}

1. Massless quarks in external magnetic field.

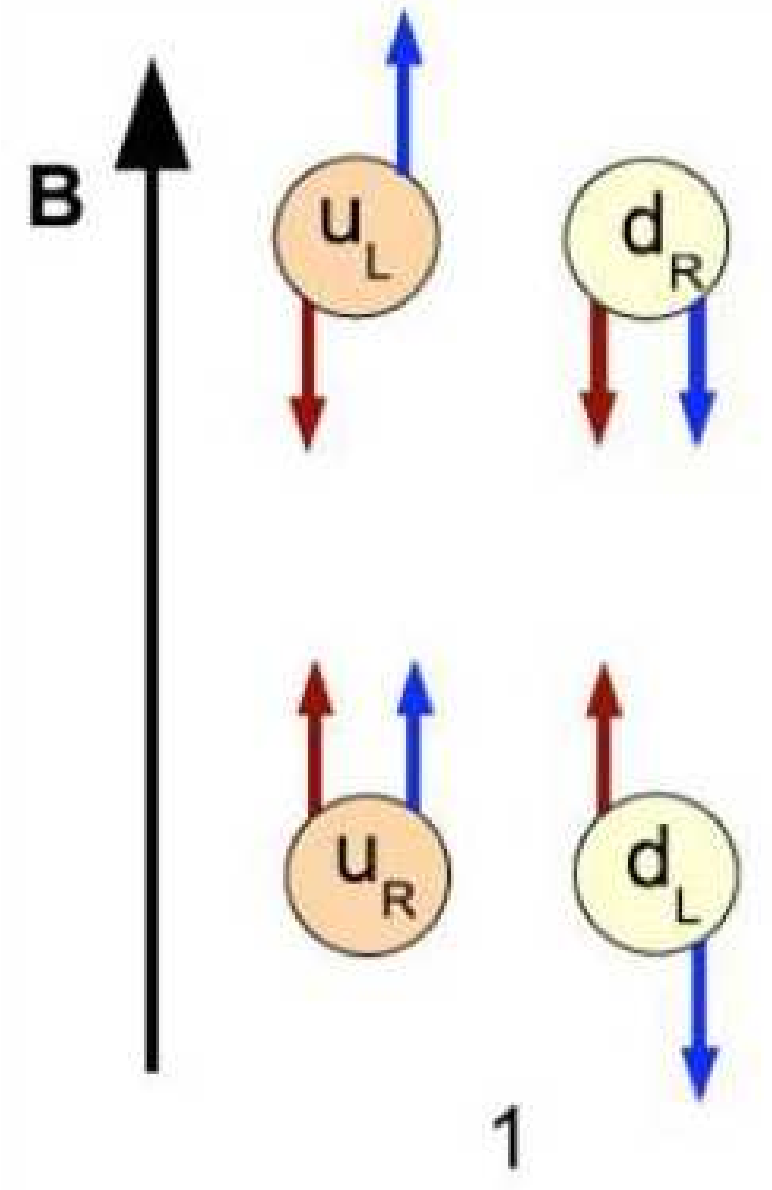

Red: momentum Blue: spin 


\section{Chiral Magnetic Effect by \\ Fukushima, Kharzeev, Warringa, McLerran}

\section{Quarks in the instatnton field.}

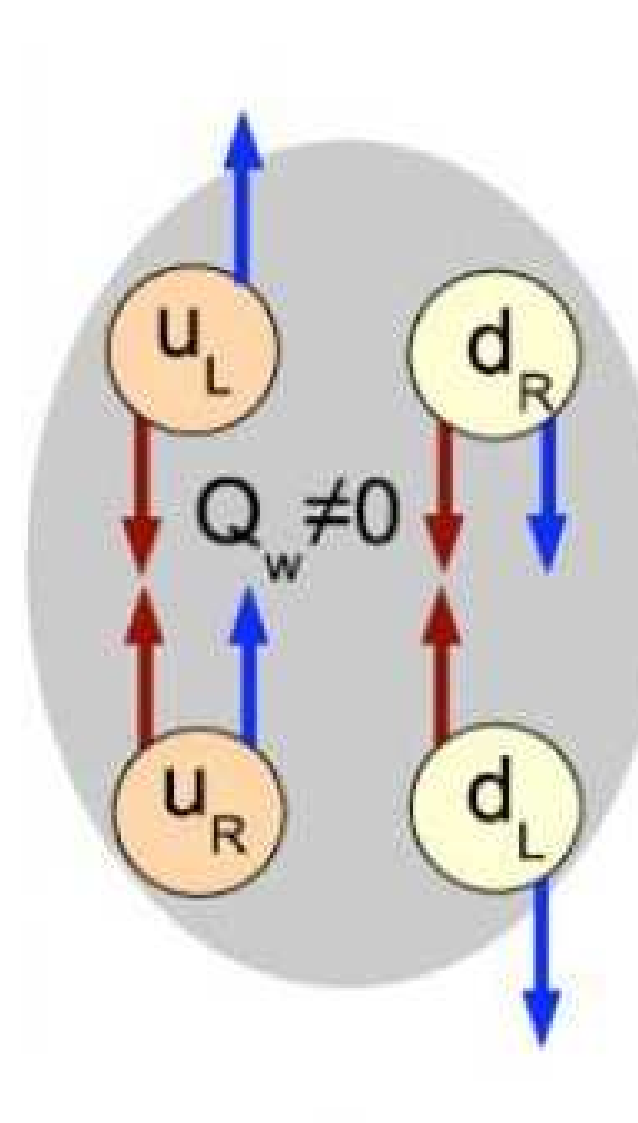

2
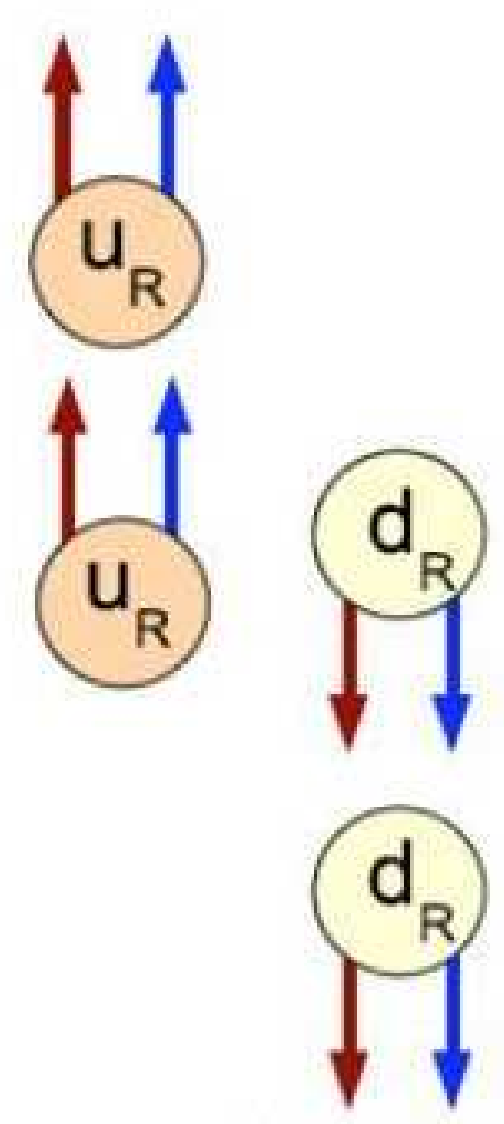

3
Red: momentum Blue: spin

Effect of topology:

$\mathrm{u}_{\mathrm{L}} \rightarrow \mathrm{u}_{\mathrm{R}}$

$d_{L} \rightarrow d_{R}$ 


\section{Chiral Magnetic Effect by \\ Fukushima, Kharzeev, Warringa, McLerran}

3. Electric current along magnetic field Red: momentum
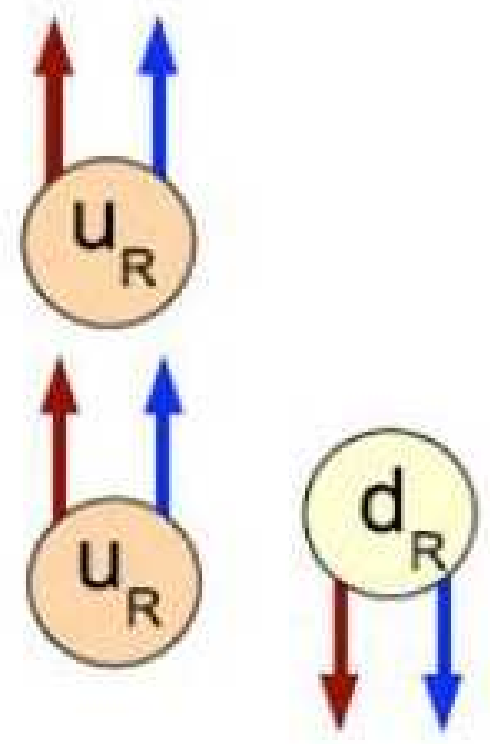

$$
\begin{aligned}
\mathrm{u}_{\mathrm{L}} & \rightarrow \mathrm{u}_{\mathrm{R}} \\
\mathrm{d}_{\mathrm{L}} & \rightarrow \mathrm{d}_{\mathrm{R}}
\end{aligned}
$$

d u-quark: $q=+2 / 3$ d-quark: $q=-1 / 3$ 


\section{Chiral Magnetic Effect by Fukushima, Kharzeev, Warringa, McLerran 3. Electric current is along magnetic field In the instanton field}

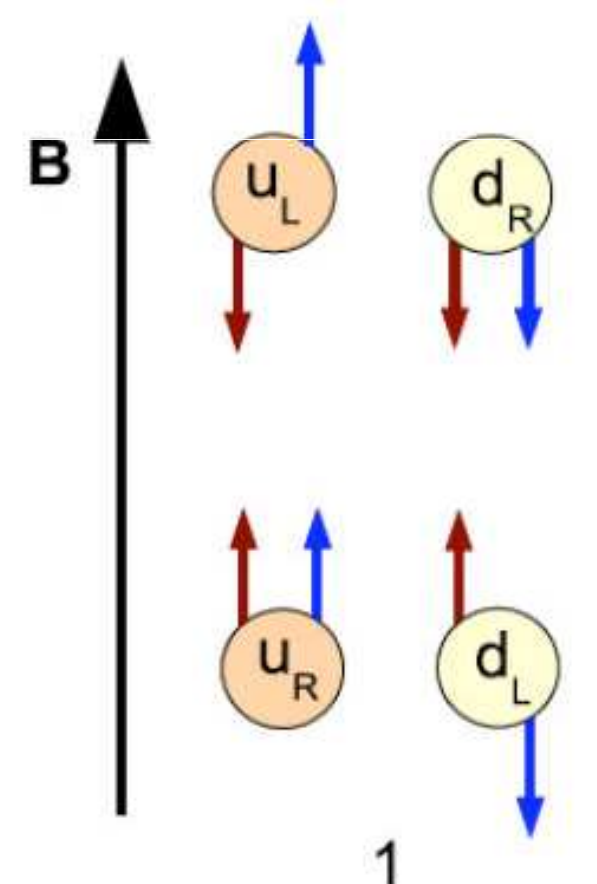

1

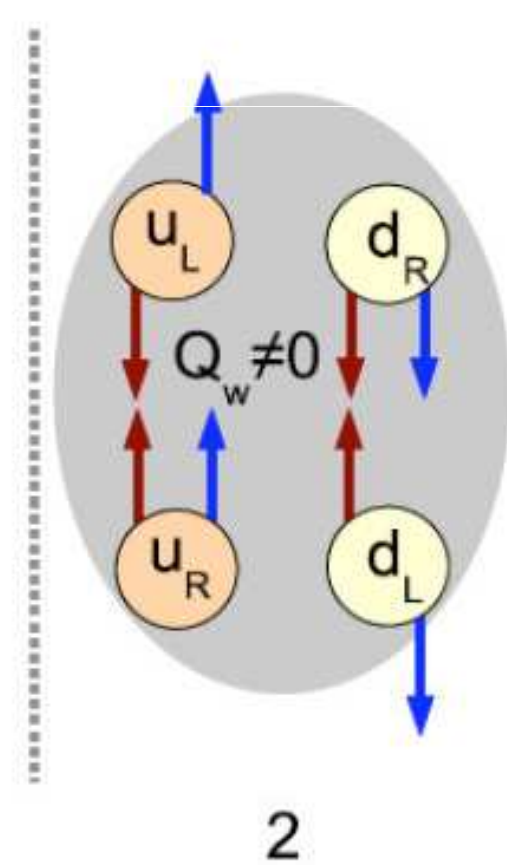

2

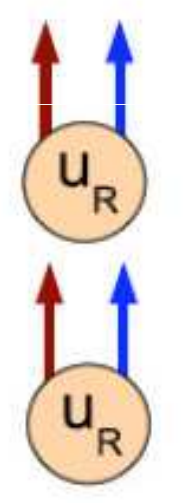

$d_{R}$ $\sqrt{d^{2}}$ 3
Red: momentum Blue: spin

Effect of topology:

$\begin{aligned} \mathbf{u}_{\mathrm{L}} & \rightarrow \mathrm{u}_{\mathbf{R}} \\ \mathbf{d}_{\mathrm{L}} & \rightarrow \mathbf{d}_{\mathbf{R}}\end{aligned}$

u-quark: $q=+2 / 3$

d-quark: $q=-1 / 3$ 


\section{D time slices of topological charge density, lattice calculations}

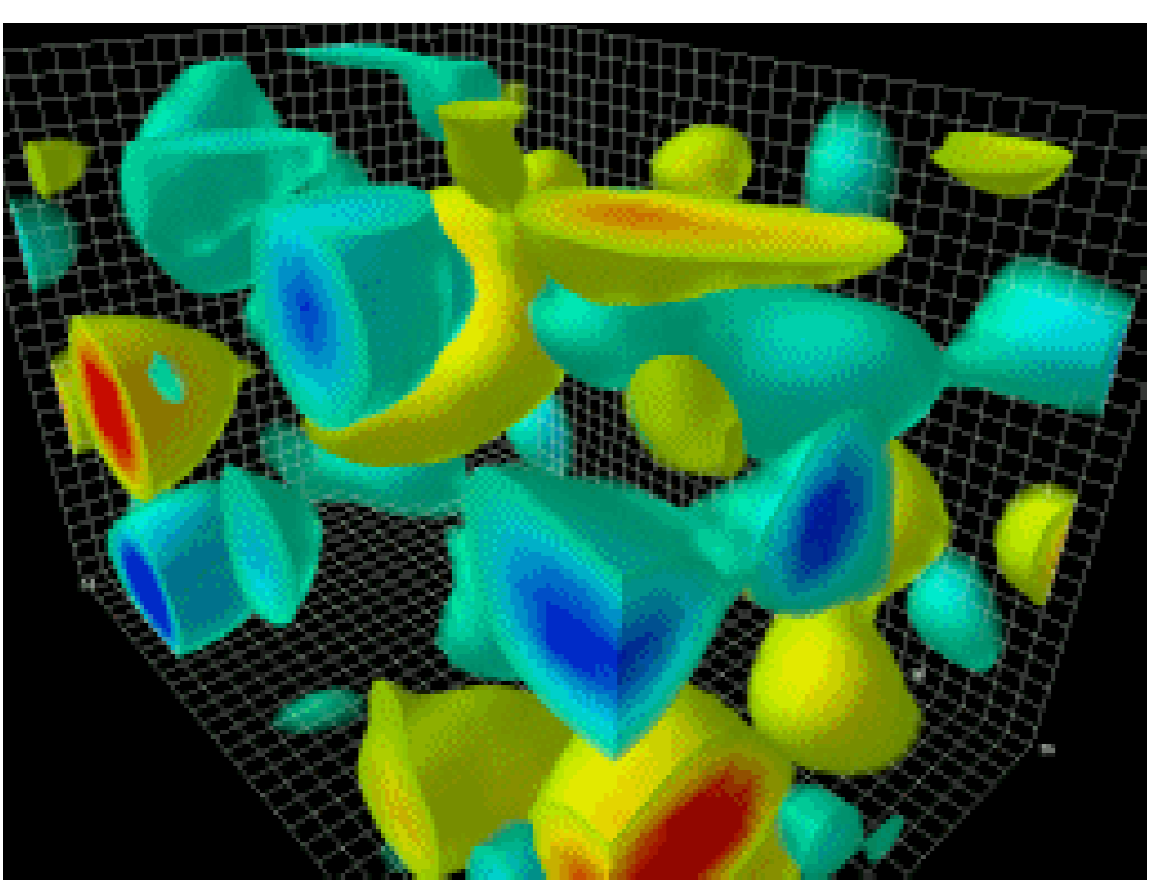

D. Leinweber

Topological charge density after vacuum cooling

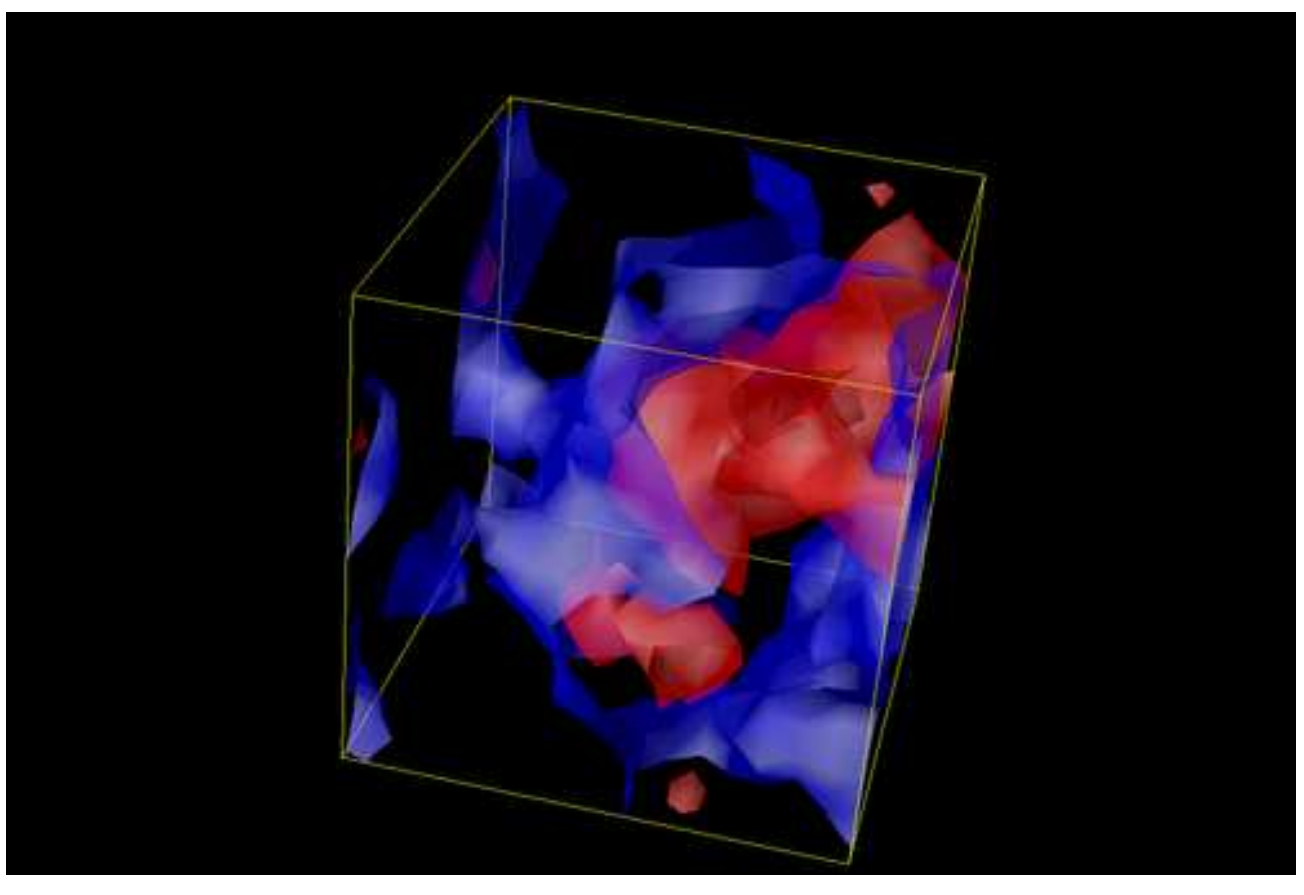

P.V.Buividovich, T.K. Kalaydzhyan, M.I. Polikarpov

Fractal topological charge density without vacuum cooling 


\section{Magnetic forces are of the order of strong interaction forces}

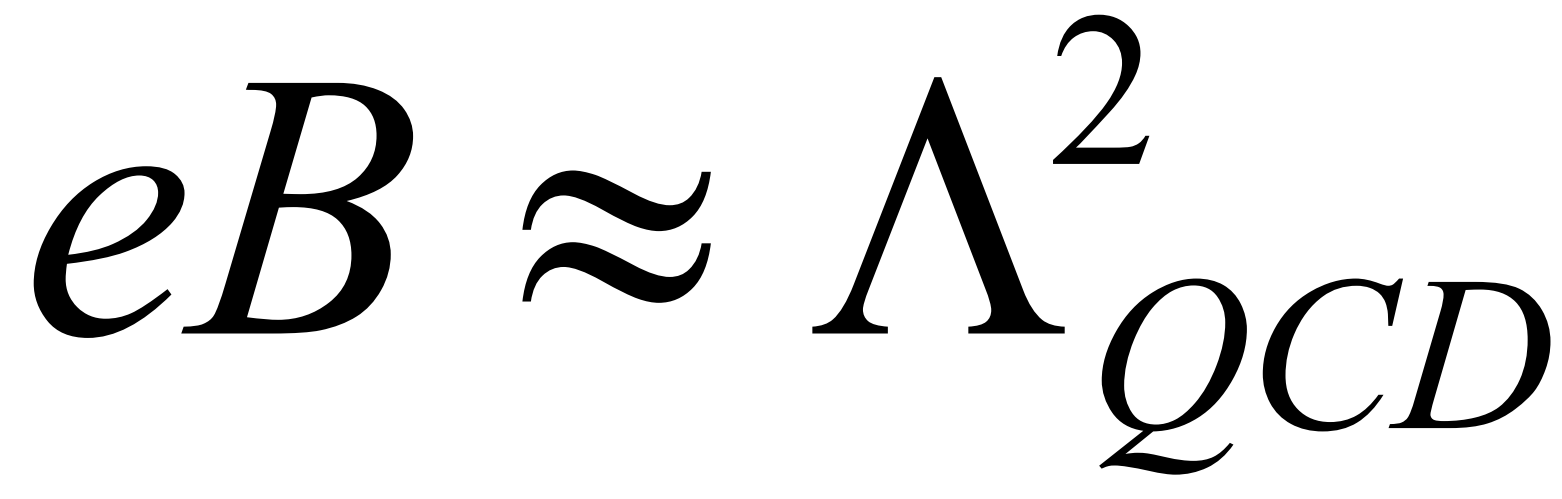

We expect the influence of magnetic field on strong interaction physics

The effects are nonperturbative,

and we use

Lattice Calculations 


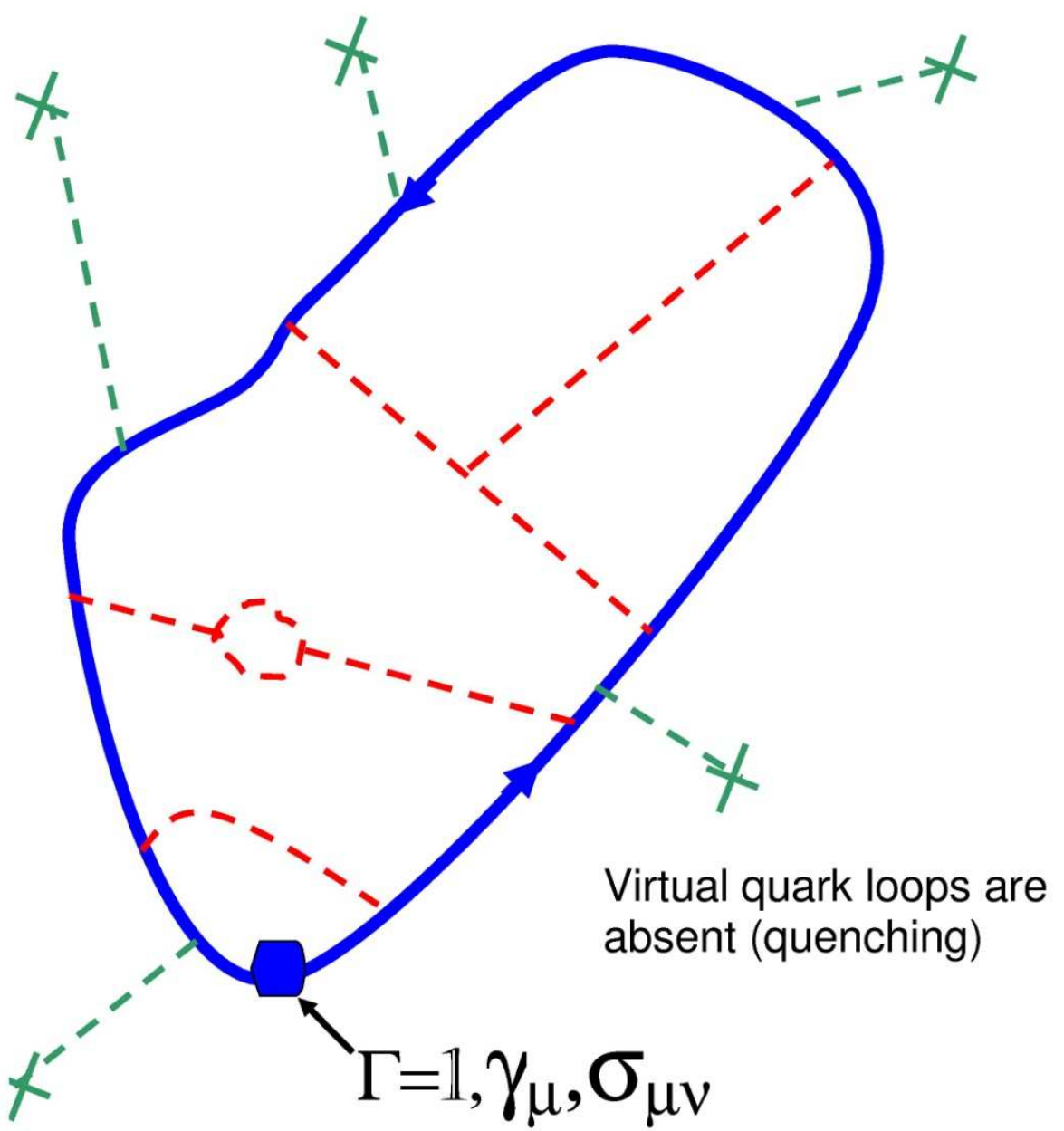

$\longrightarrow$ External quark

- - - - $\quad$ Virtual gluon

- - - - - Photon (external magnetic field)
We calculate $<\bar{\psi} \Gamma \psi>; \Gamma=1, \gamma_{\mu}, \sigma_{\mu \nu}$ in the external magnetic field and in the presence of the vacuum gluon fields We consider SU(2) gauge fields and quenched approximation

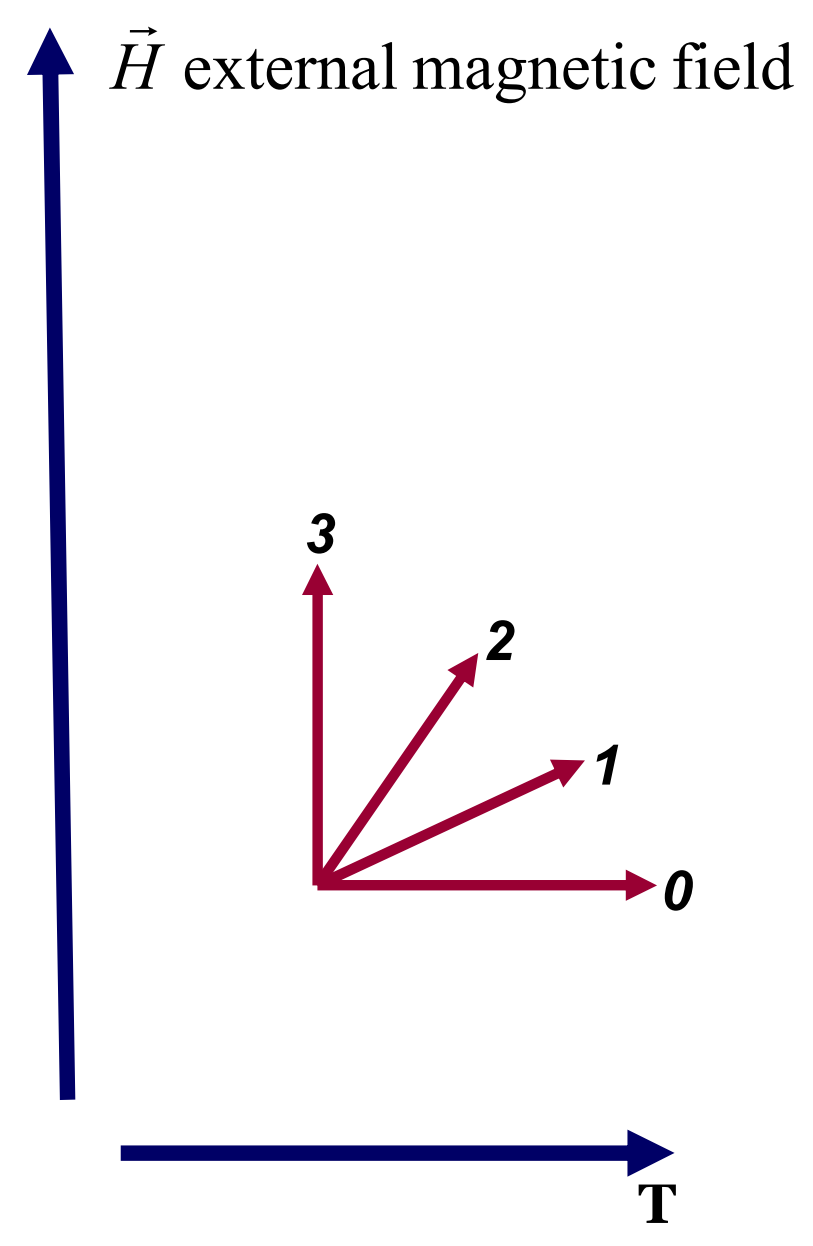




\section{Quenched vacuum, overlap Dirac operator, external magnetic field}

$$
e B=\frac{2 \pi q k}{L^{2}} ; e B \geq(250 \mathrm{Mev})^{2}
$$


Density of the electric charge vs. magnetic field, 3D time slices

$$
B=0
$$

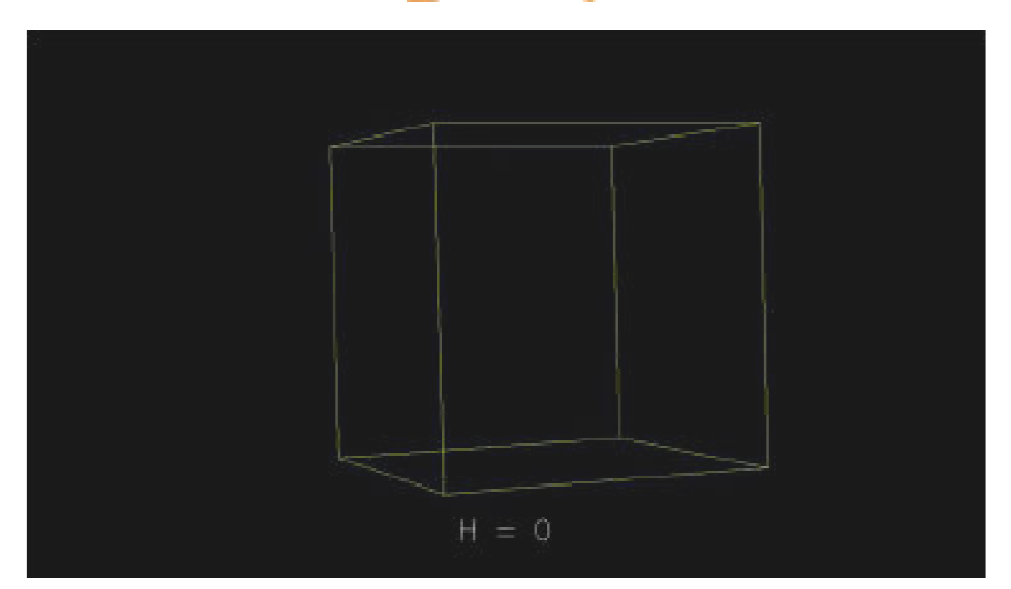

$$
B=(780 \mathrm{MeV})^{2}
$$

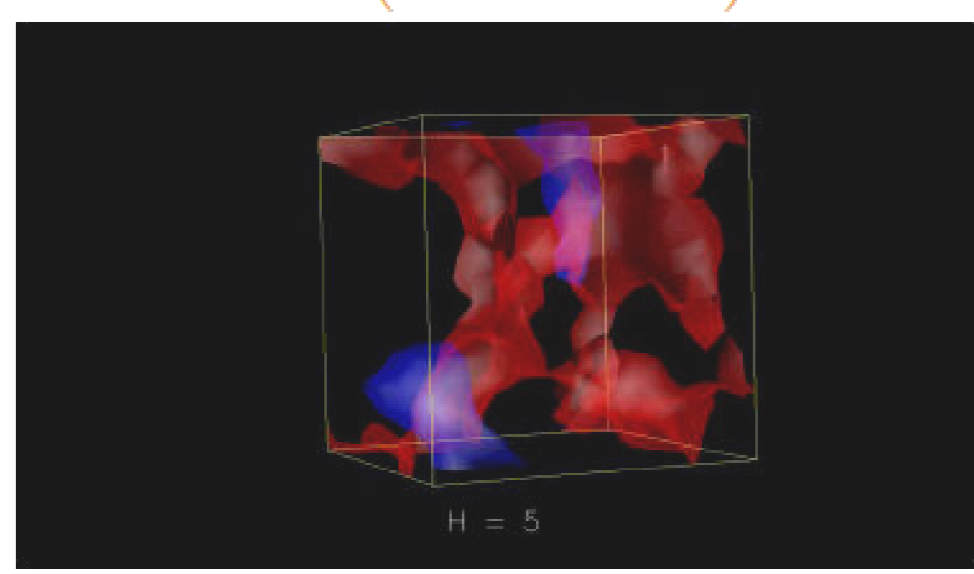

$B=(500 \mathrm{MeV})^{2}$

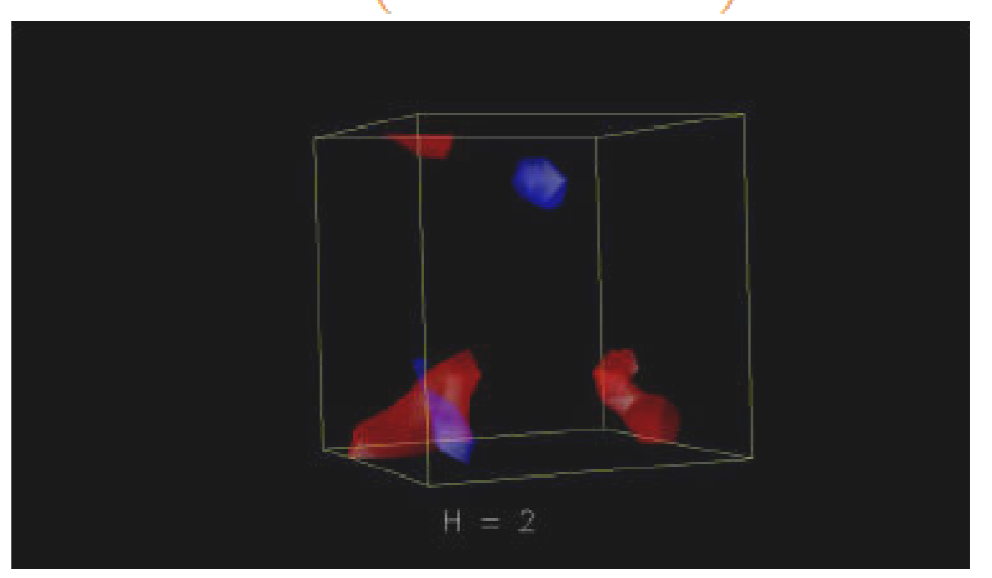

$$
B=(1.1 \mathrm{GeV})^{2}
$$

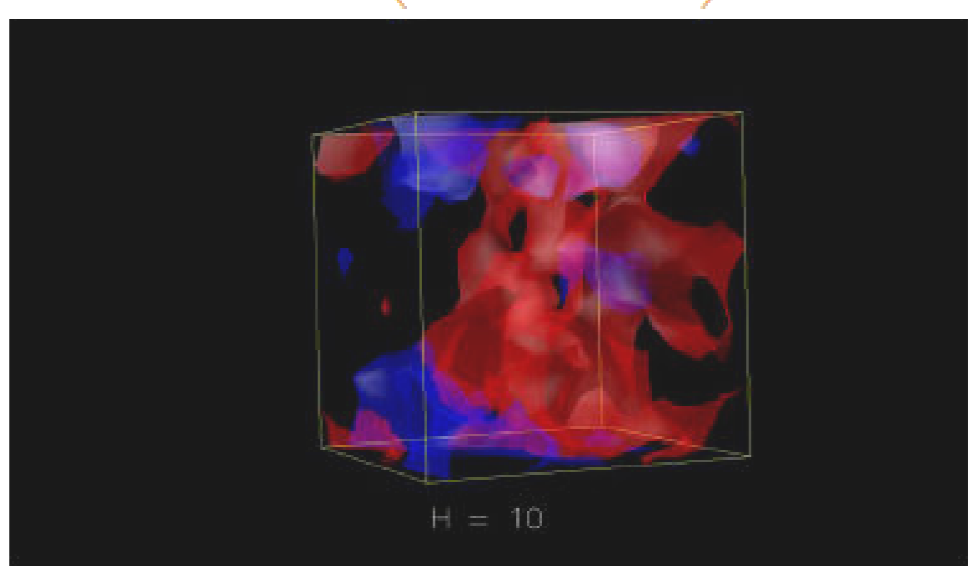


1. Chiral Magnetic Effect on the lattice, numerical results $\mathrm{T}=0$

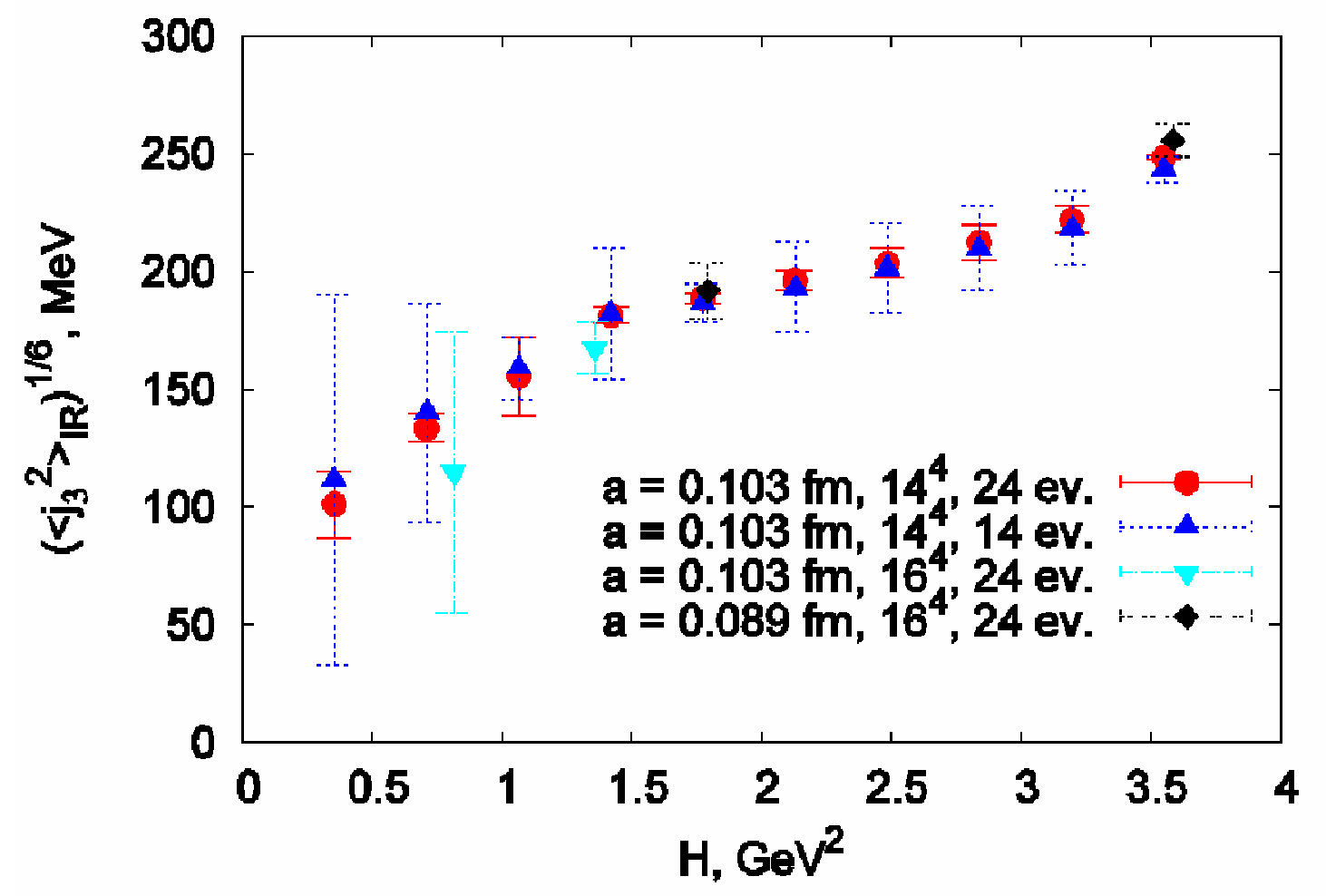

Regularized electric current:

$<j_{3}^{2}>_{I R}=<j_{3}^{2}(H, T)>-<j_{3}^{2}(0,0)>, \quad j_{3}=\bar{\psi} \gamma_{3} \psi$ 
Chiral Magnetic Effect on the lattice, numerical comparison of results nearTc and nearzero

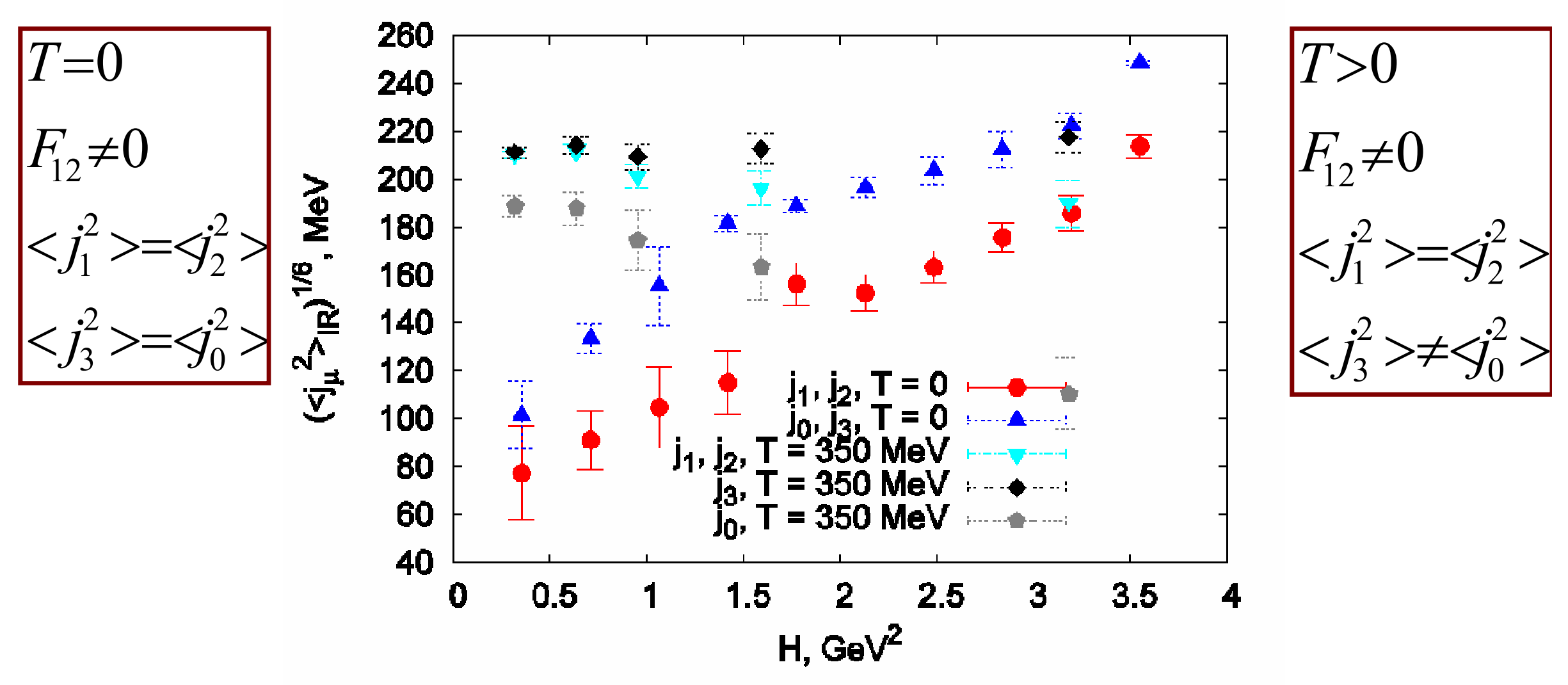

Regularized electric current:

$$
<j_{i}^{2}>_{I R}=<j_{i}^{2}(H, T)>-<j_{i}^{2}(0,0)>, \quad j_{i}=\bar{\psi} \gamma_{i} \psi
$$




\section{Chiral Magnetic Effect, EXPERIMENT VS LATTICE DATA $(\mathrm{Au}+\mathrm{Au})$}

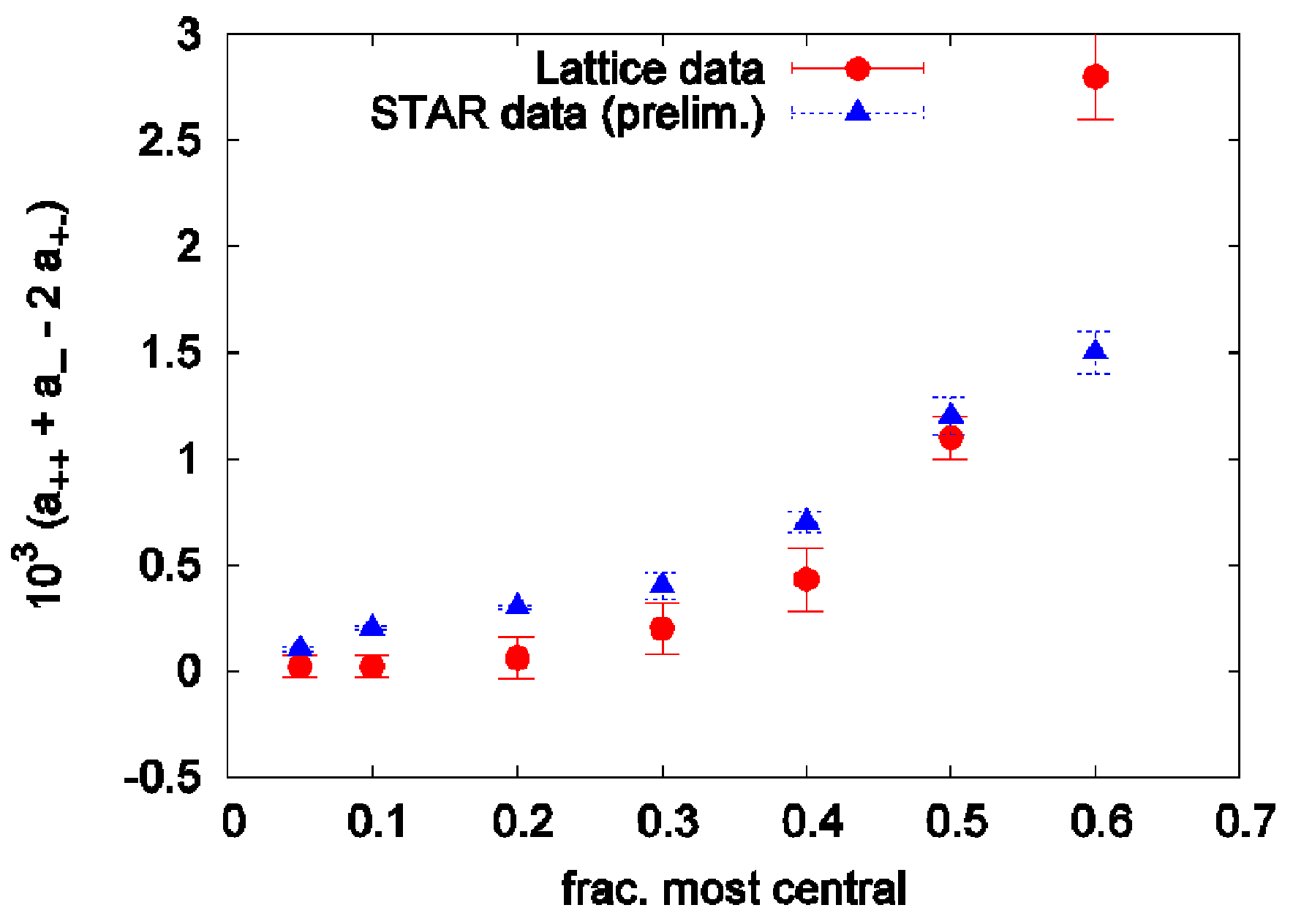




\section{Chiral Magnetic Effect, EXPERIMENT VS LATTICE DATA}

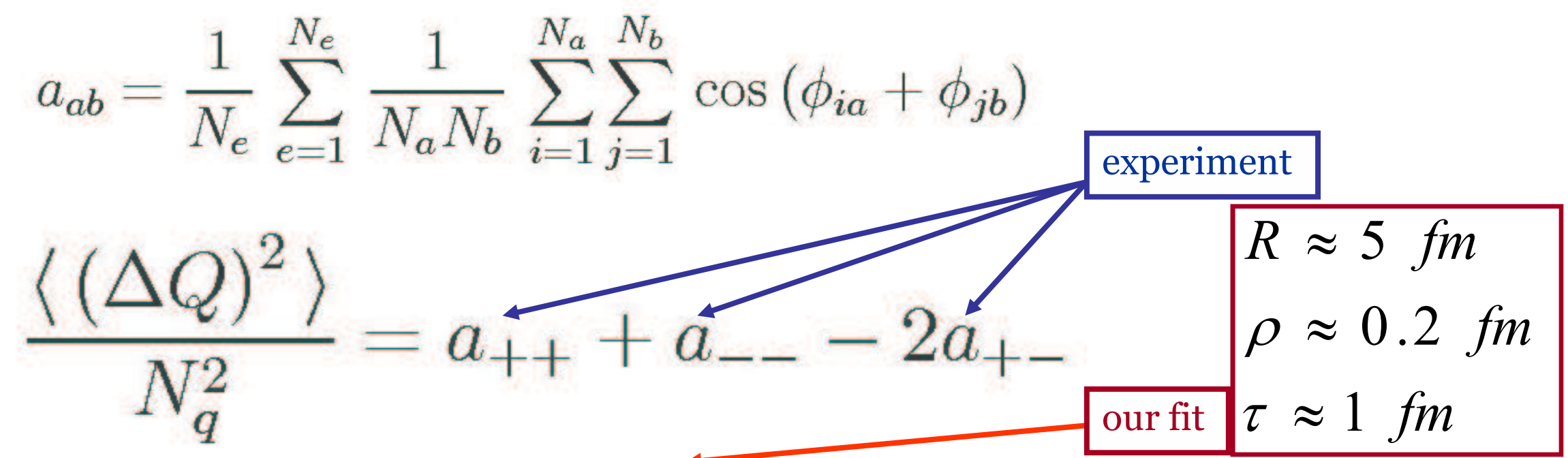

D. E. Kharzeev,

L. D. McLerran, and

H. J. Warringa, Nucl. Phys. A 803, 227 (2008),
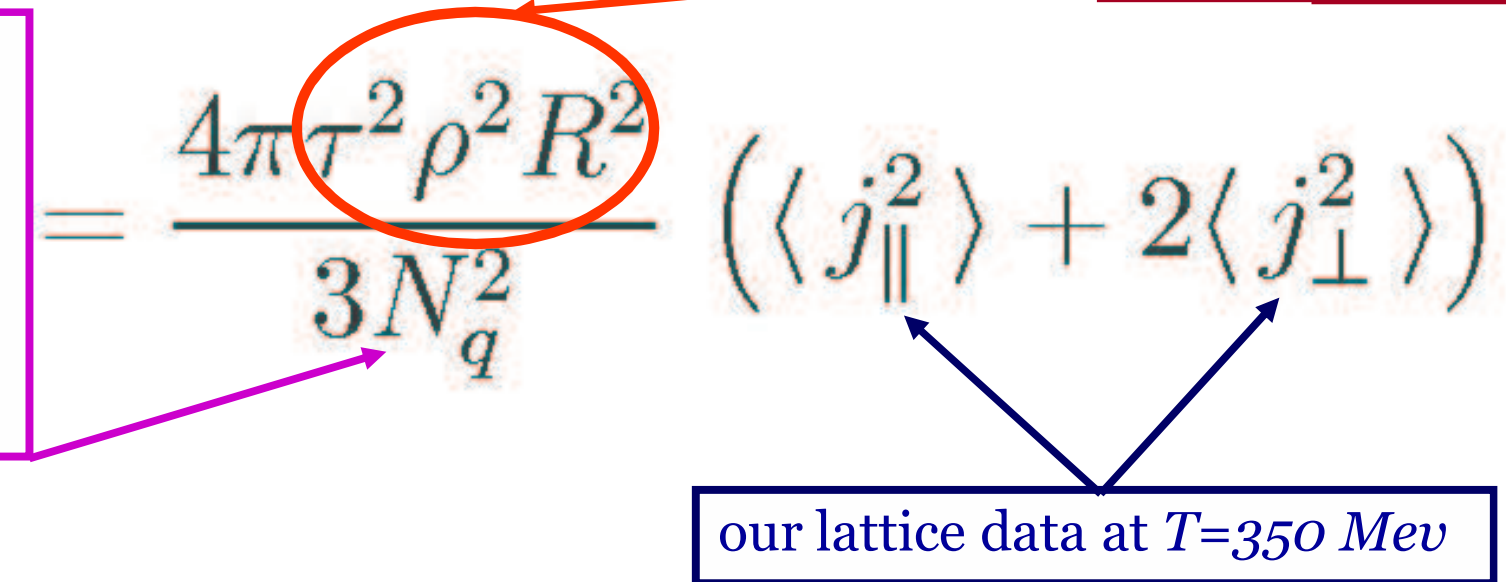
1.2 Magnetic Field Induced Conductivity of the Vacuum

Qualitative definition of conductivity, $\sigma$

$$
\begin{gathered}
<j_{\mu}(x) j_{\nu}(y)>=C+A \cdot \frac{\exp \{-m|x-y|\}}{r^{\alpha}} \\
j_{\mu}(x)=\bar{q}(x) \gamma_{\mu} q(x) \\
\sigma \propto C
\end{gathered}
$$




\section{Magnetic Field Induced Conductivity of the Vacuum}

$$
\begin{aligned}
& \sigma_{i j}=\frac{\rho_{i j}(0)}{4 T} \quad \text { - Conductivity (Kubo formula) } \\
& G_{i j}(\tau)=\int_{0}^{+\infty} \frac{d w}{2 \pi} K(w, \tau) \rho_{i j}(w), \text { Maximal entropy method } \\
& K(w, \tau)=\frac{w}{2 T} \frac{\cosh \left(w\left(\tau-\frac{1}{2 T}\right)\right)}{\sinh \left(\frac{w}{2 T}\right)}, \\
& G_{i j}(\tau)=\int d^{3} \vec{x}\left\langle j_{i}(\overrightarrow{0}, 0) j_{j}(\vec{x}, \tau)\right\rangle
\end{aligned}
$$




\section{Magnetic Field Induced Conductivity of the Vacuum}

$$
\sigma_{i j}=\frac{\rho_{i j}(0)}{4 T} \quad \text { - Conductivity (Kubo formula) }
$$

For weak constant electric field

$$
<j_{i}>=\sigma_{i k} E_{k}
$$




\section{Magnetic Field Induced Conductivity of} the Vacuum

\section{Calculations in SU(2) gluodynamics}

$\left\langle\bar{q}(x) \gamma_{i} q(x) \bar{q}(y) \gamma_{j} q(y)\right\rangle$

$=\int \mathcal{D} A_{\mu} e^{-S_{Y M}\left[A_{\mu}\right]} \operatorname{Tr}\left(\frac{1}{\mathcal{D}+m} \gamma_{i} \frac{1}{\mathcal{D}+m} \gamma_{j}\right)$

We use overlap operator + Shifted Unitary Minimal Residue Method (Borici and Allcoci (2006)) to obtain fermion propagator

$$
G_{i j}(\tau)=\int d^{3} \vec{x}\left\langle j_{i}(\overrightarrow{0}, 0) j_{j}(\vec{x}, \tau)\right\rangle
$$




\section{Magnetic Field Induced Conductivity of the Vacuum Calculations in SU(2) gluodynamics}

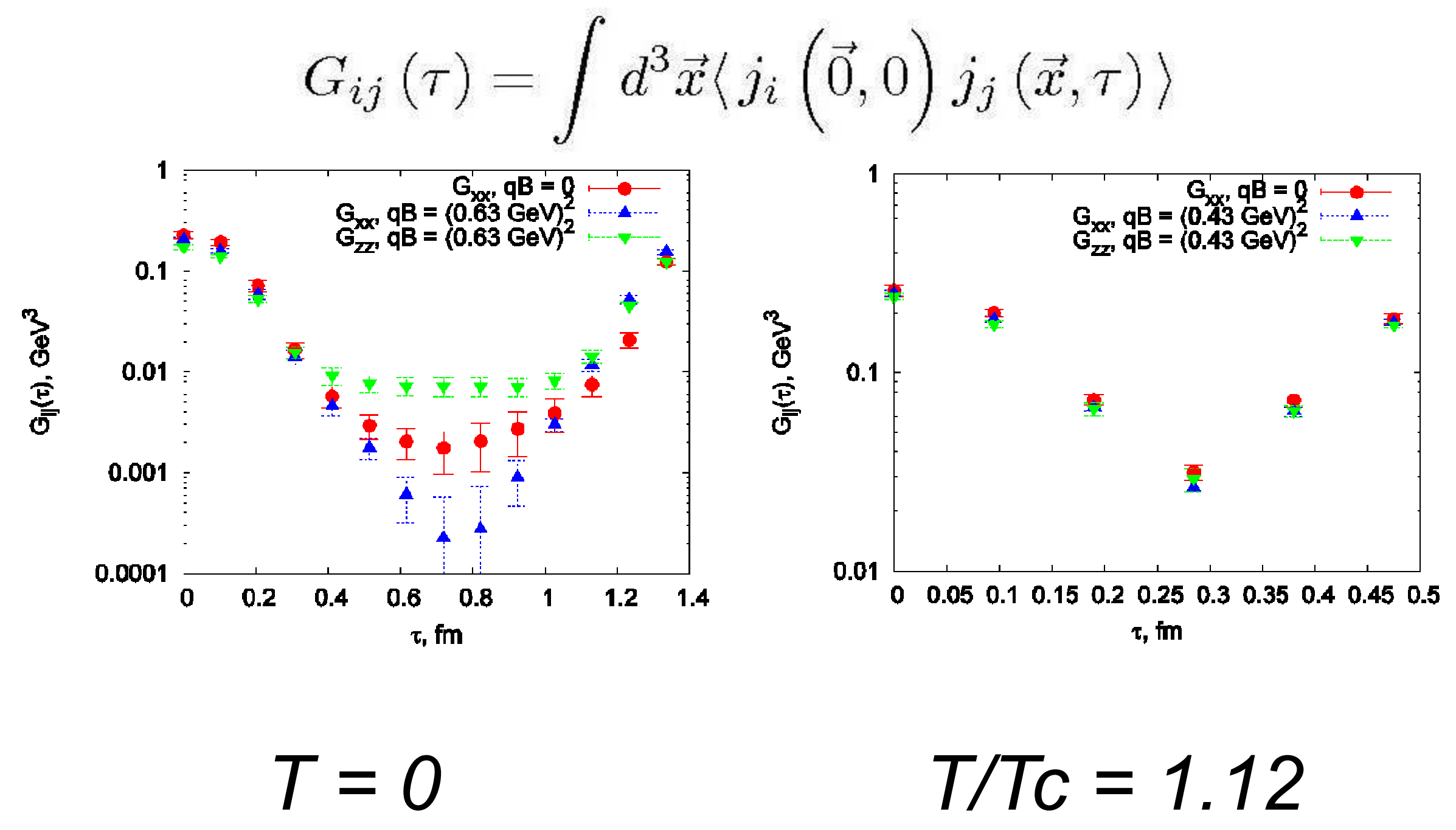


Calculations in SU(2) gluodynamics, conductivity along magnetic field at

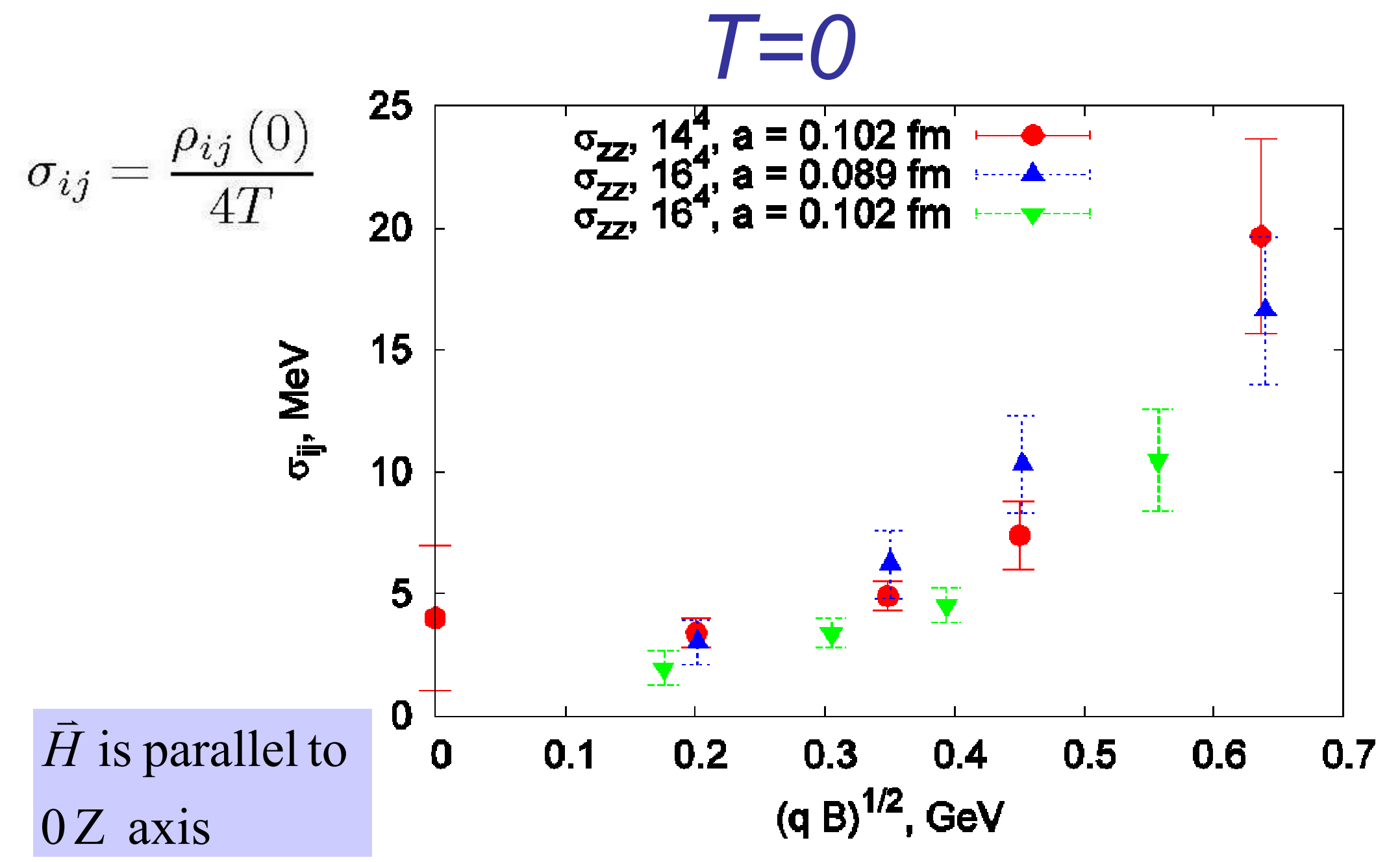




\section{Calculations in SU(2) gluodynamics, conductivity along magnetic field at $T=0$}

$\sigma_{i j}=\frac{\rho_{i j}(0)}{4 T}$ At $T=0, B=0$ vacuum is insulator
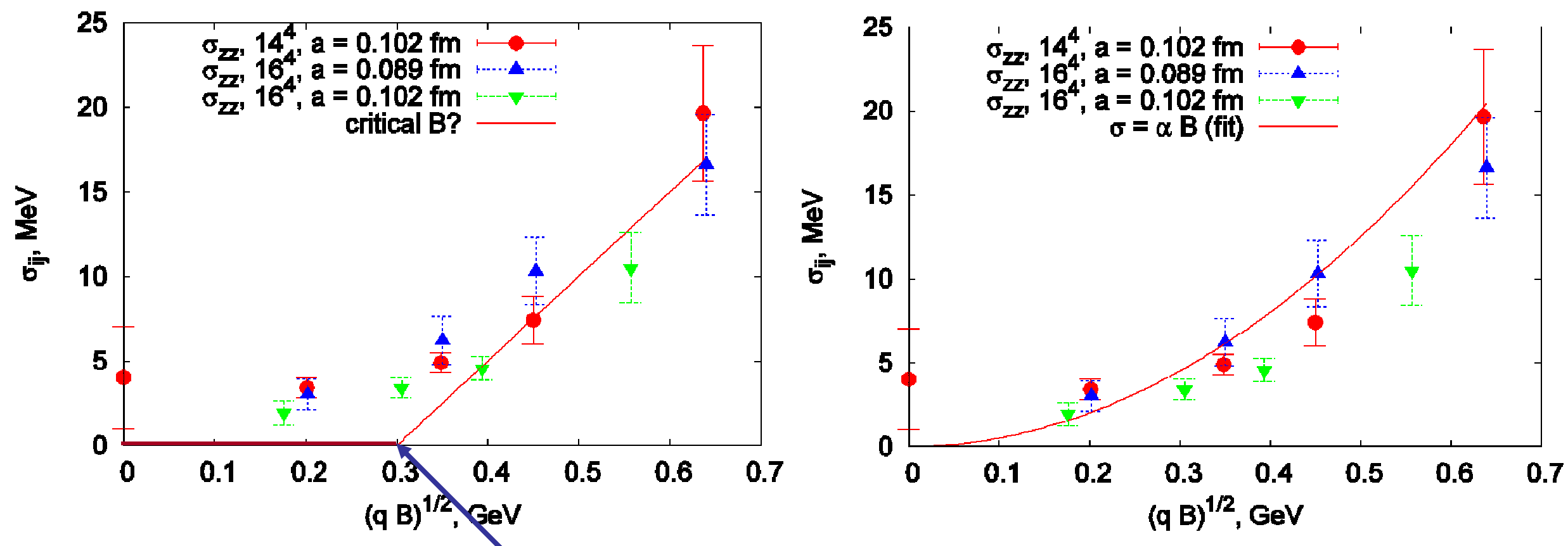

Critical value of magnetic field? 
Calculations in SU(2) gluodynamics, conductivity along magnetic field at $T=0, T>0$

$\sigma_{i j}=\frac{\rho_{i j}(0)}{4 T}$

$\vec{H}$ is parallel to $0 \mathrm{Z}$ axis

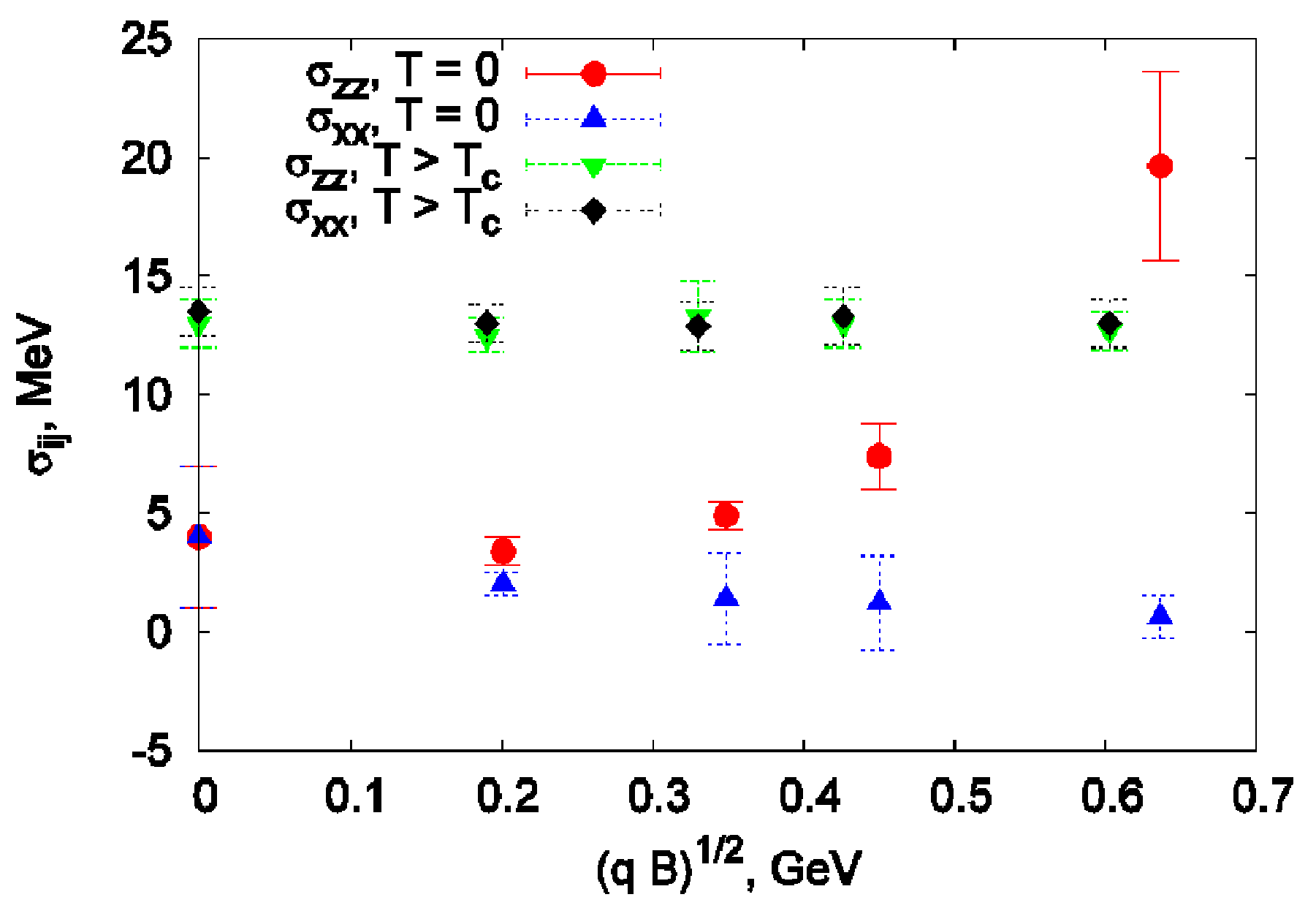




\section{Calculations in $\mathrm{SU}(2)$ gluodynamics, conductivity at $T=0$, variation of the quark mass}

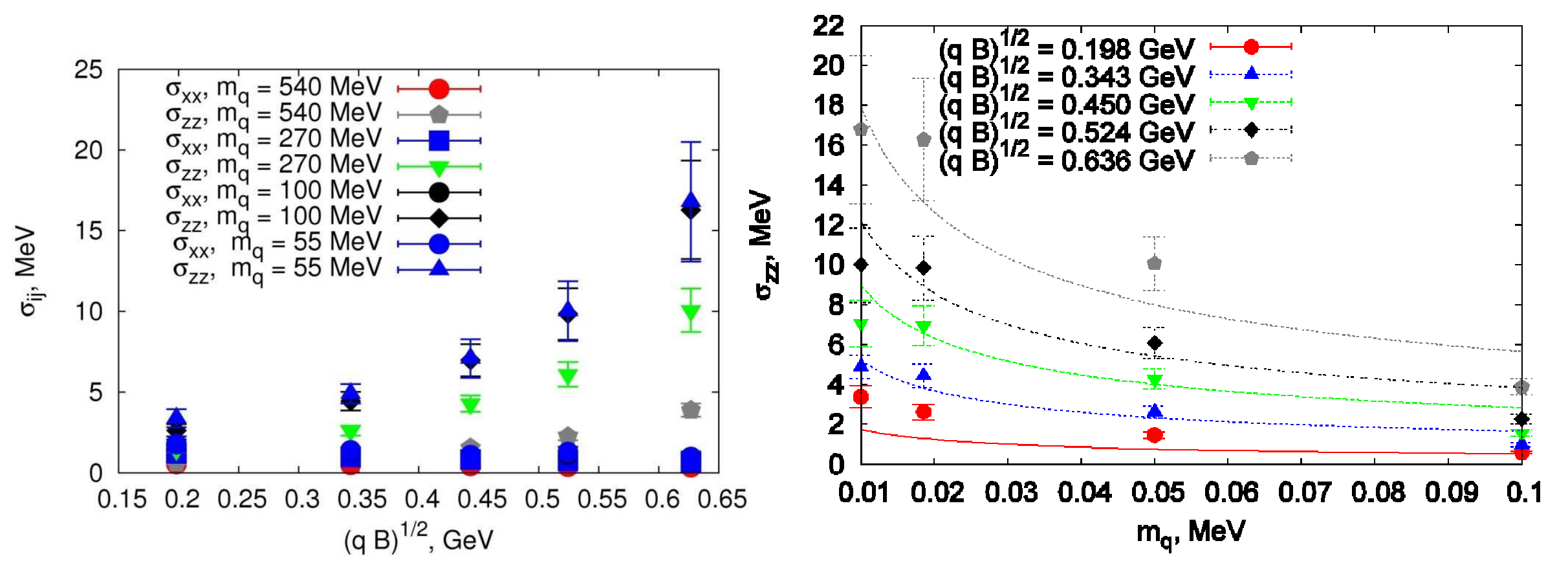

$$
\begin{array}{r}
\sigma_{z z}\left(m_{q}, q B\right) \sim m_{q}^{-\alpha}(|q B|)^{\beta} \quad \alpha=(0.45 \pm 0.06) \\
\beta=(1.1 \pm 0.2)
\end{array}
$$




\section{Calculations in $\mathrm{SU}(2)$ gluodynamics, conductivity at $T=0$, variation of the quark mass and magnetic field}

$$
\sigma_{i j} \propto \frac{B_{i} B_{j}}{B \sqrt{m_{q}}}
$$
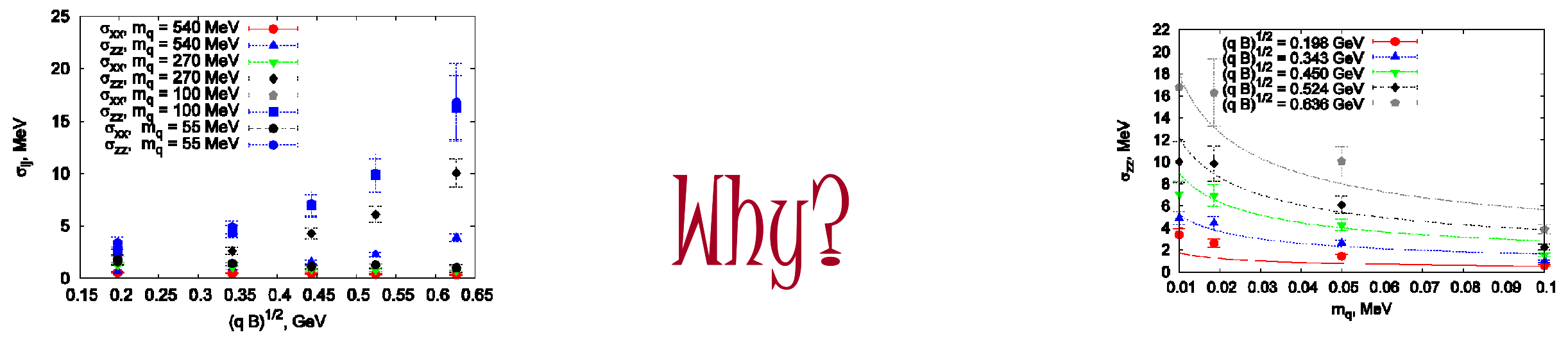


\subsection{Dilepton emission rate}

L. D. McLerran and T. Toimela, Phys. Rev. D 31, 545 (1985),

E. L. Bratkovskaya, O. V. Teryaev, and V. D.Toneev, Phys. Lett. B 348, 283 (1995)

$$
\frac{R}{V}=-4 e^{4} \int \frac{d^{3} p_{1}}{(2 \pi)^{3} 2 E_{1}} \frac{d^{3} p_{1}}{(2 \pi)^{3} 2 E_{1}} L^{\mu \nu}\left(p_{1}, p_{2}\right) \frac{\rho_{\mu \nu}(q)}{q^{4}},(7)
$$

where $p_{1}$ and $p_{2}$ are the momenta of the leptons, $q=p_{1}+p_{2}, m$ is their mass and $L^{\mu \nu}=$ $\left(\left(p_{1} \cdot p_{2}+m^{2}\right) \eta^{\mu \nu}-p_{1}^{\mu} p_{2}^{\nu}-p_{2}^{\mu} p_{1}^{\nu}\right)$ is the dilepton tensor. If the electric conductivity is nonzero in the direction of the magnetic field, for sufficiently small $p_{1}, p_{2}$ one has $\rho_{i j}(q) \approx \rho_{i j}(0) \sim \sigma_{i j} \sim B_{i} B_{j} /|B|$, and hence

$$
\sigma_{i j}=\frac{\rho_{i j}(0)}{4 T}
$$

$$
\frac{R}{V} \sim \int \frac{d^{3} p_{1}}{(2 \pi)^{3} 2 E_{1}} \frac{d^{3} p_{1}}{(2 \pi)^{3} 2 E_{1}} \frac{\left(p_{1} \cdot B\right)\left(p_{2} \cdot B\right)}{|B|} .
$$


- There should be more soft dileptons in the direction of magnetic field
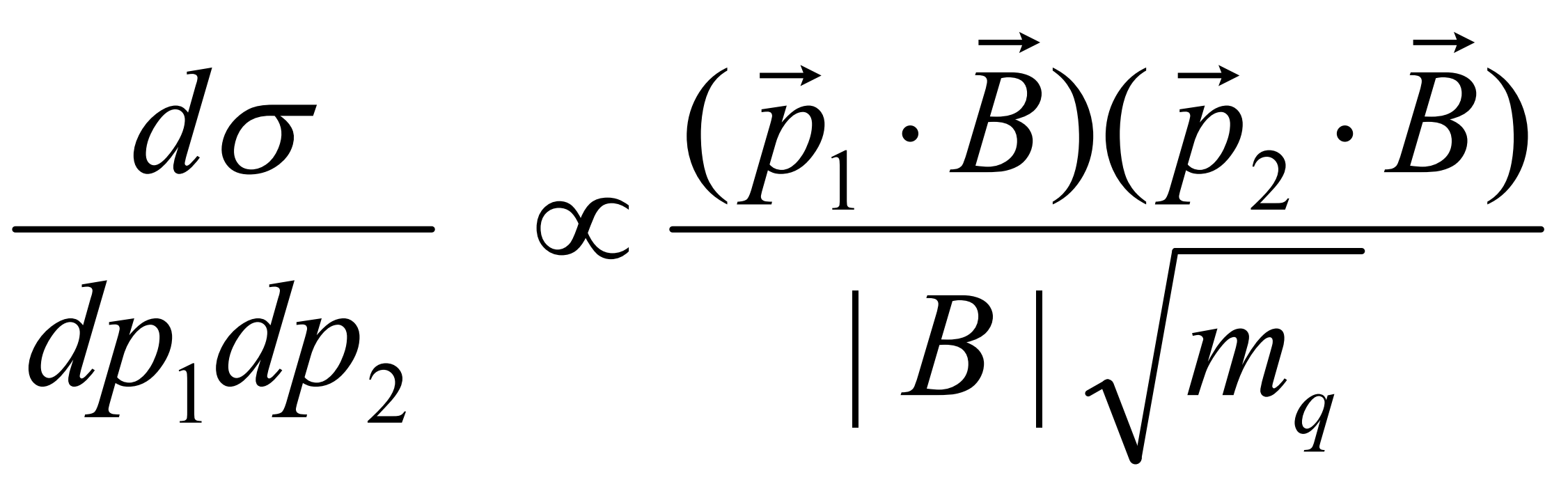


\section{Other effects induced by magnetic field}

2.1 Chiral symmetry breaking

2.2 Magnetization of the vacuum

2.3 Electric dipole moment of quark along the direction of the magnetic field 
3. Chiral condensate in QCD

$$
\Sigma=-\langle\bar{\psi} \psi>
$$

$$
m_{\pi}^{2} f_{\pi}^{2}=m_{q}<\bar{\psi} \psi>
$$




\section{Chiral condensate vs. field strength, SU(2) gluodynamics}
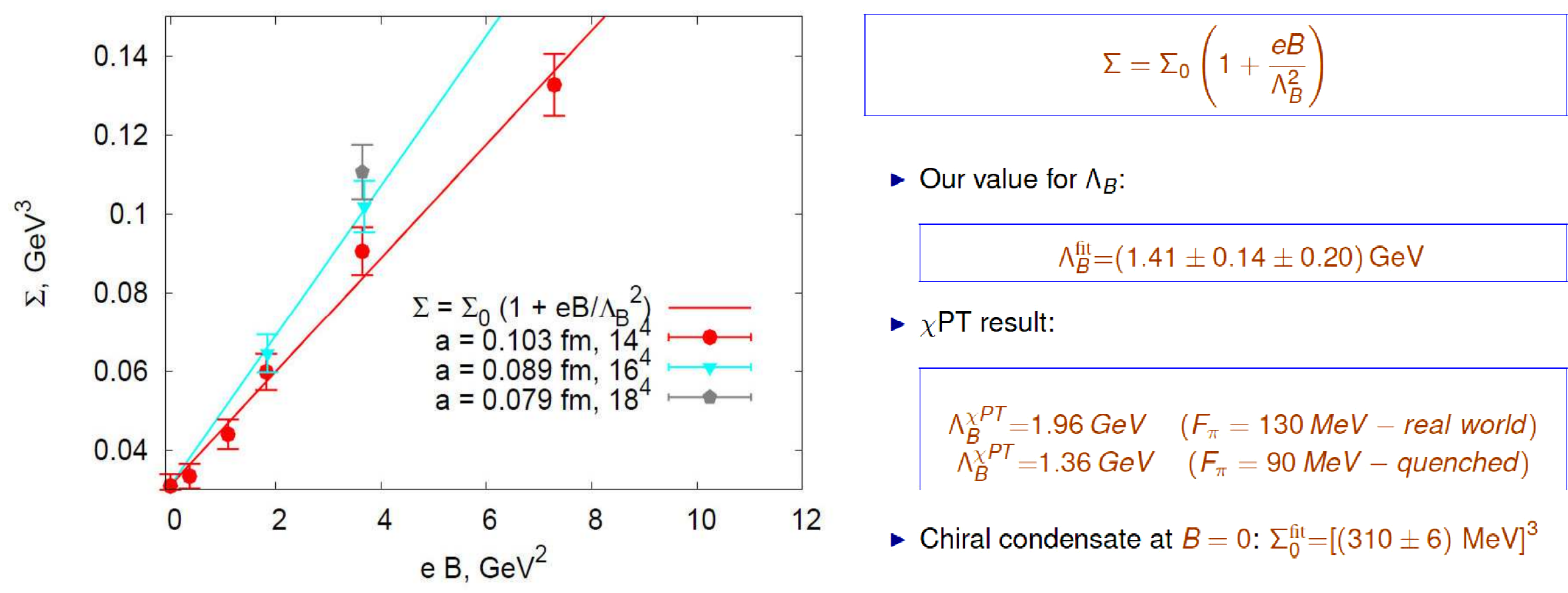

- Our value for $\Lambda_{B}$ :

$\Lambda_{B}^{\mathrm{fit}}=(1.41 \pm 0.14 \pm 0.20) \mathrm{GeV}$

- $\chi^{\text {PT result: }}$

$$
\begin{array}{cc}
\Lambda_{B}^{\chi P T}=1.96 \mathrm{GeV} & \left(F_{\pi}=130 \mathrm{MeV}-\text { real world }\right) \\
\Lambda_{B}^{\chi P T}=1.36 \mathrm{GeV} & \left(F_{\pi}=90 \mathrm{MeV}-\text { quenched }\right)
\end{array}
$$

- Chiral condensate at $B=0: \Sigma_{0}^{\mathrm{fit}}=[(310 \pm 6) \mathrm{MeV}]^{3}$

We are in agreement with the chiral perturbation theory: the chiral condensate is a linear function of the strength of the magnetic field! 


\section{Chiral condensate vs. field strength, SU(3) gluodynamics}

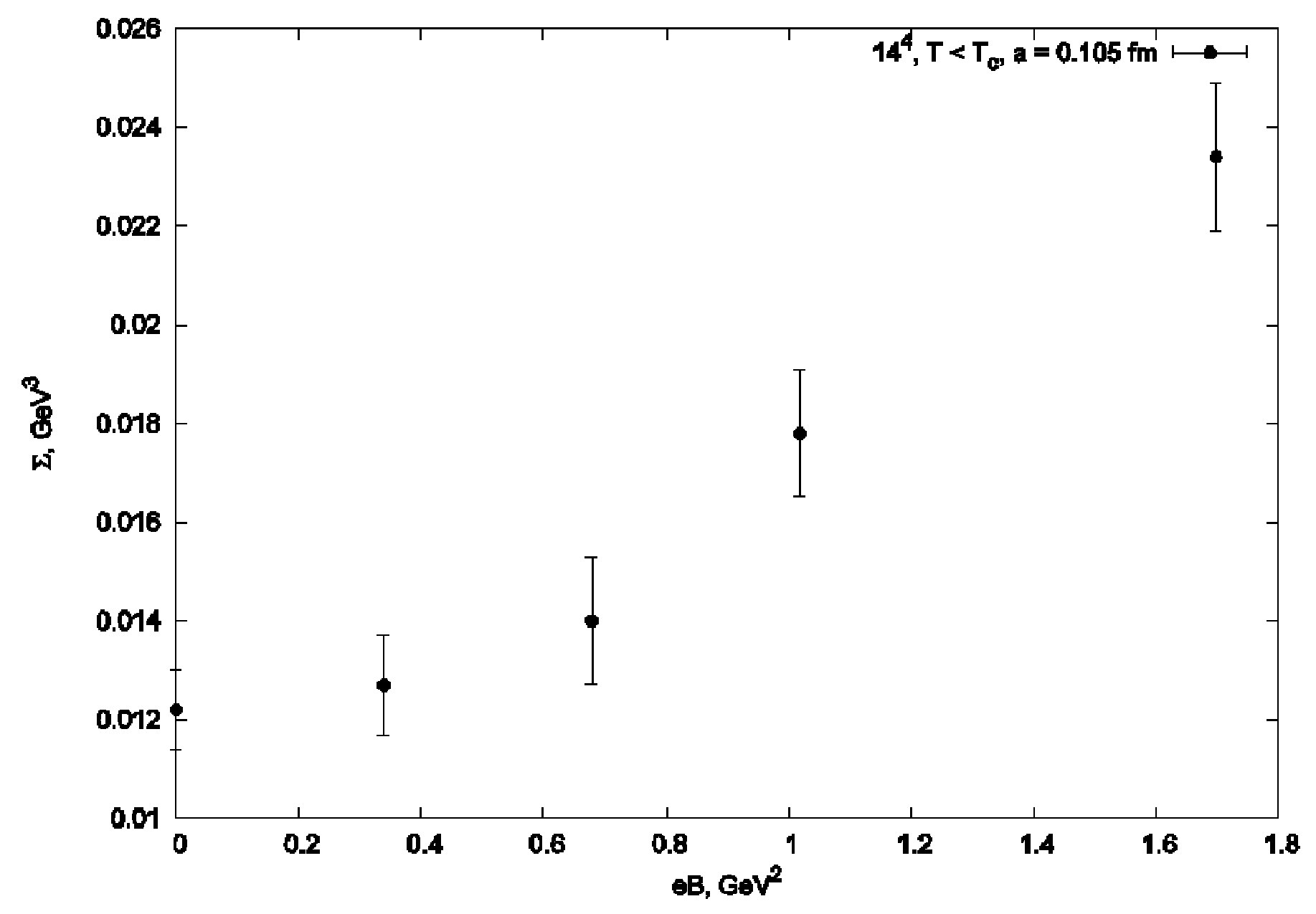




\section{Localization of Dirac Eigenmodes}

Typical densities of the nearzero eigenmodes vs. the strength of the external magnetic field
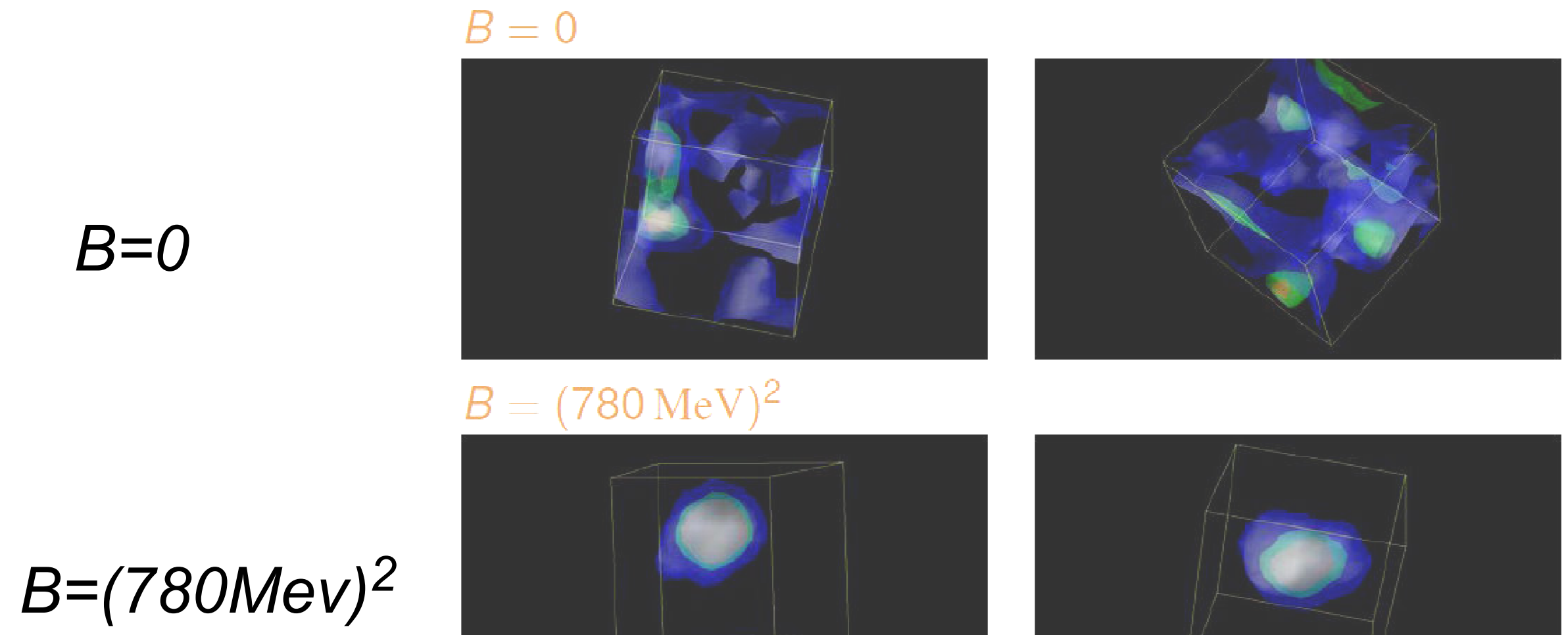

$B=(780 \mathrm{MeV})^{2}$
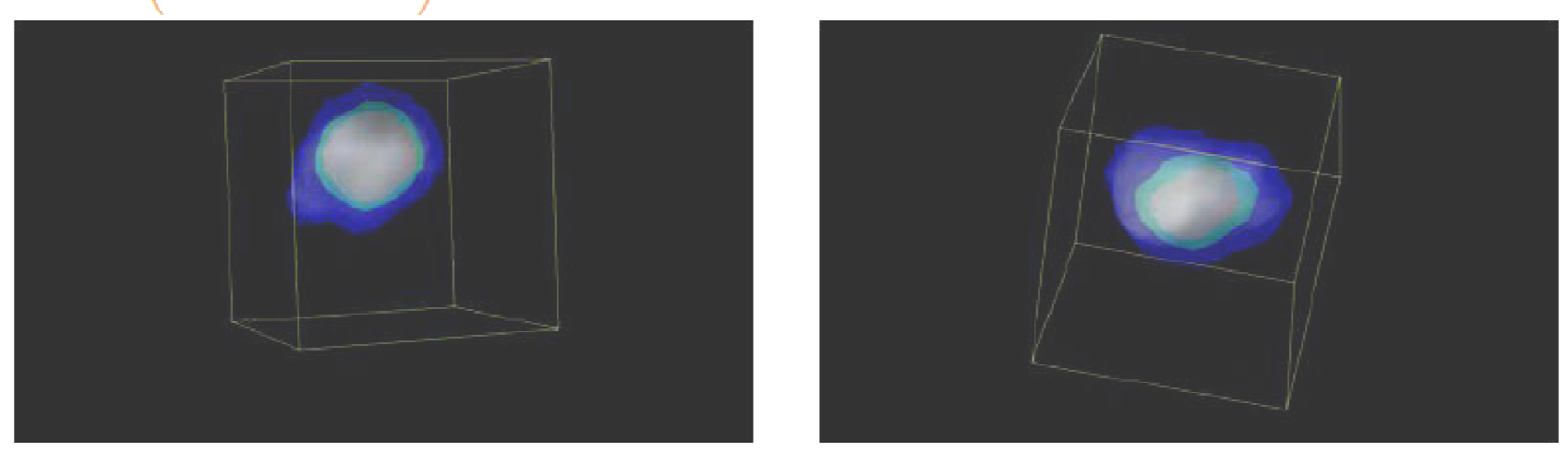


\section{Magnetization of the vacuum as a function of the magnetic field}

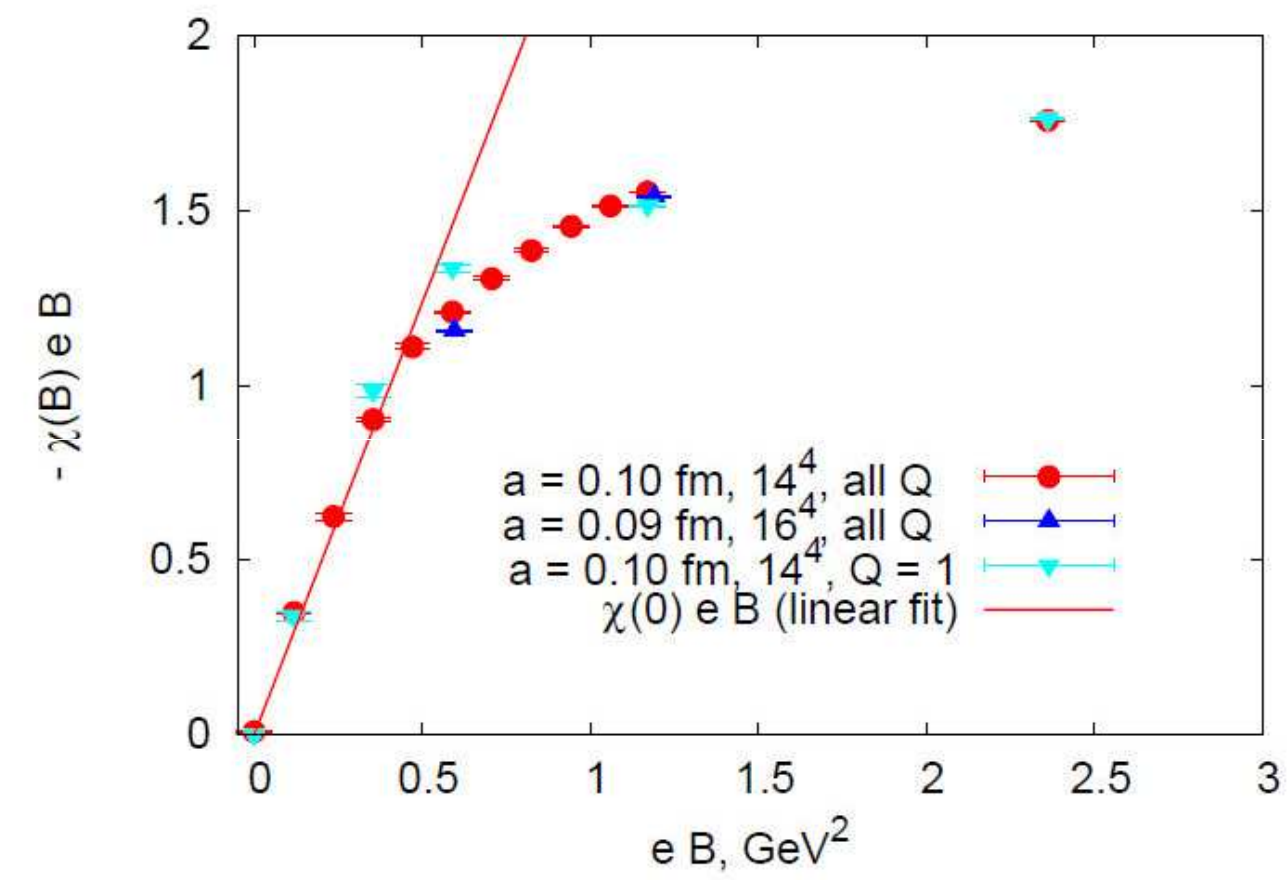

Spins of virtual quarks turn parallel to the magnetic field

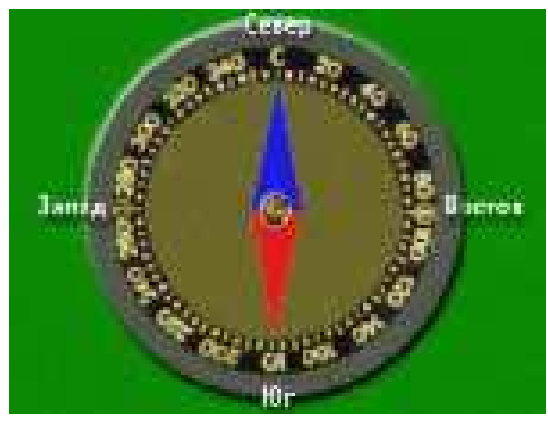

$$
\begin{aligned}
& \left\langle\bar{\psi} \sigma_{\alpha \beta} \psi>=\chi<\bar{\psi} \psi>F_{\alpha \beta}\right. \\
& \sigma_{\alpha \beta}=\frac{1}{2 i}\left[\gamma_{\alpha}, \gamma_{\beta}\right]
\end{aligned}
$$

$\langle\bar{\psi} \psi>\chi=-46(3) M e v \leftrightarrow$ our result $<\bar{\psi} \psi>\chi \approx-50 \mathrm{Mev} \leftrightarrow \mathrm{QCD}$ sum rules (I. I. Balitsky, 1985, P. Ball, 2003.) 


\section{Generation of the anomalous quark electric dipole moment along the axis of magnetic field}

M Large correlation between square of the electric dipole moment

$$
\sigma_{0 i}=i \bar{\psi}\left[\gamma_{0}, \gamma_{i}\right] \psi \quad \text { and chirality } \quad \rho_{5}=\bar{\psi} \gamma_{5} \psi
$$

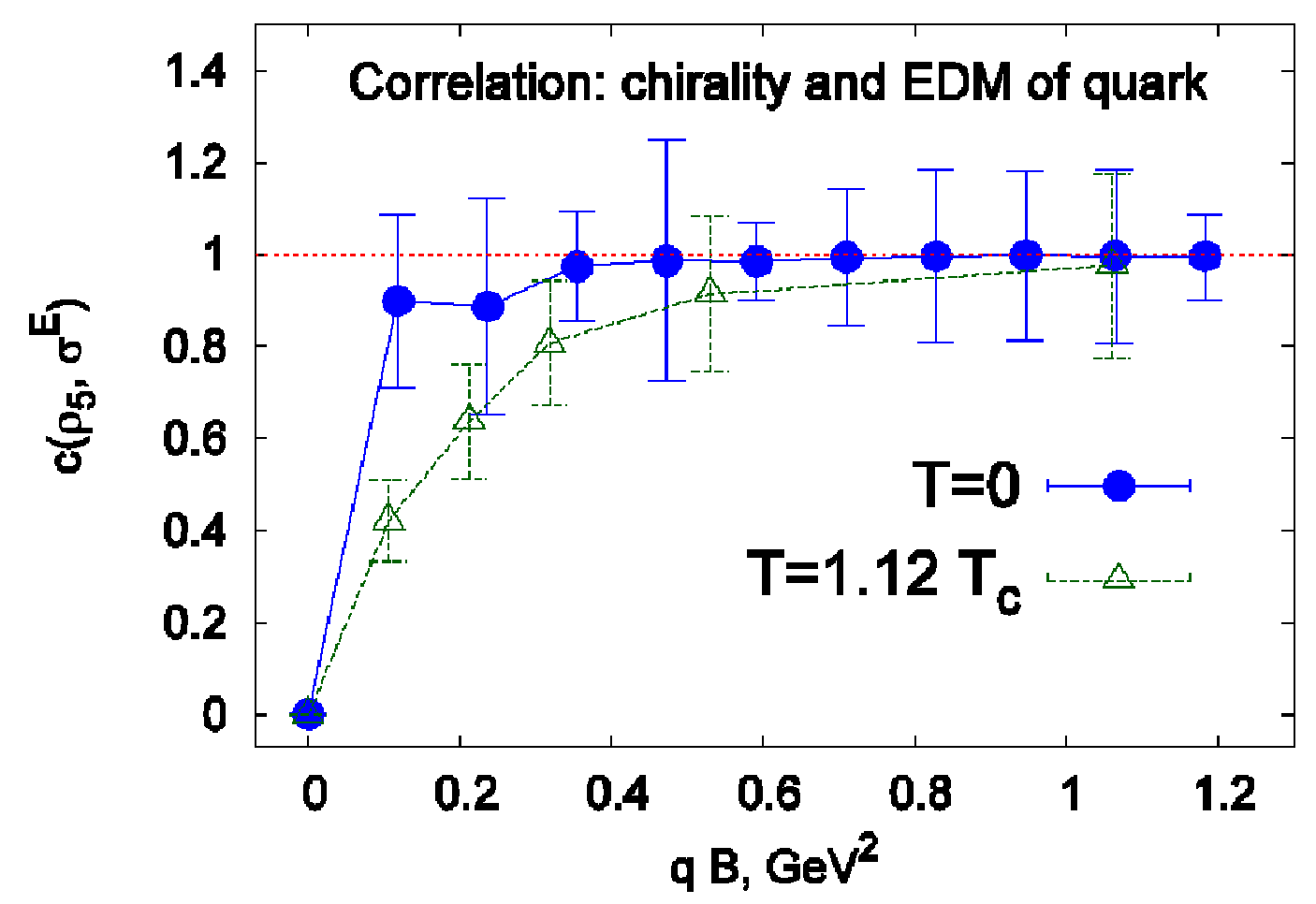




\section{Electric currents in instanton field+magnetic field (CME)}

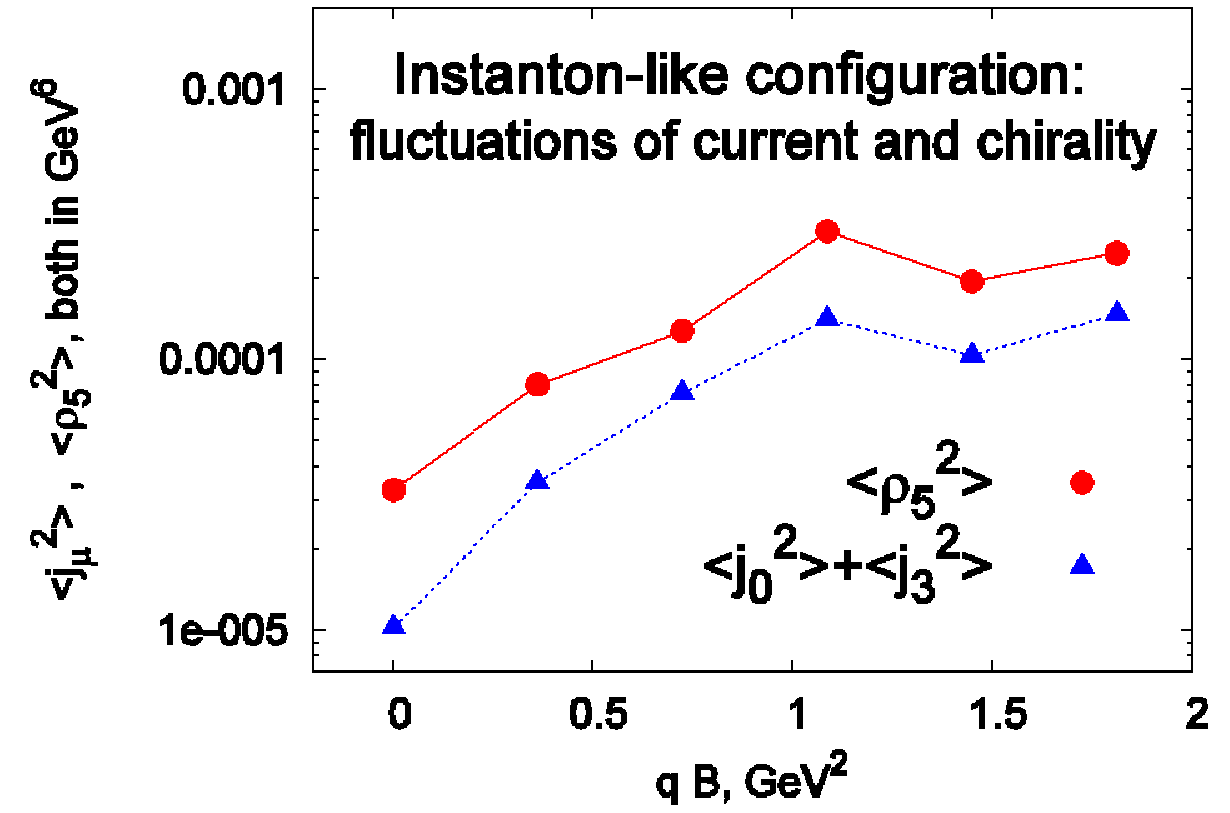

The fluctuations of the chirality $\rho_{5}=\bar{\psi} \gamma_{5} \psi$ and the fluctuations of the longitudinal electric current as a function of the magnetic field.

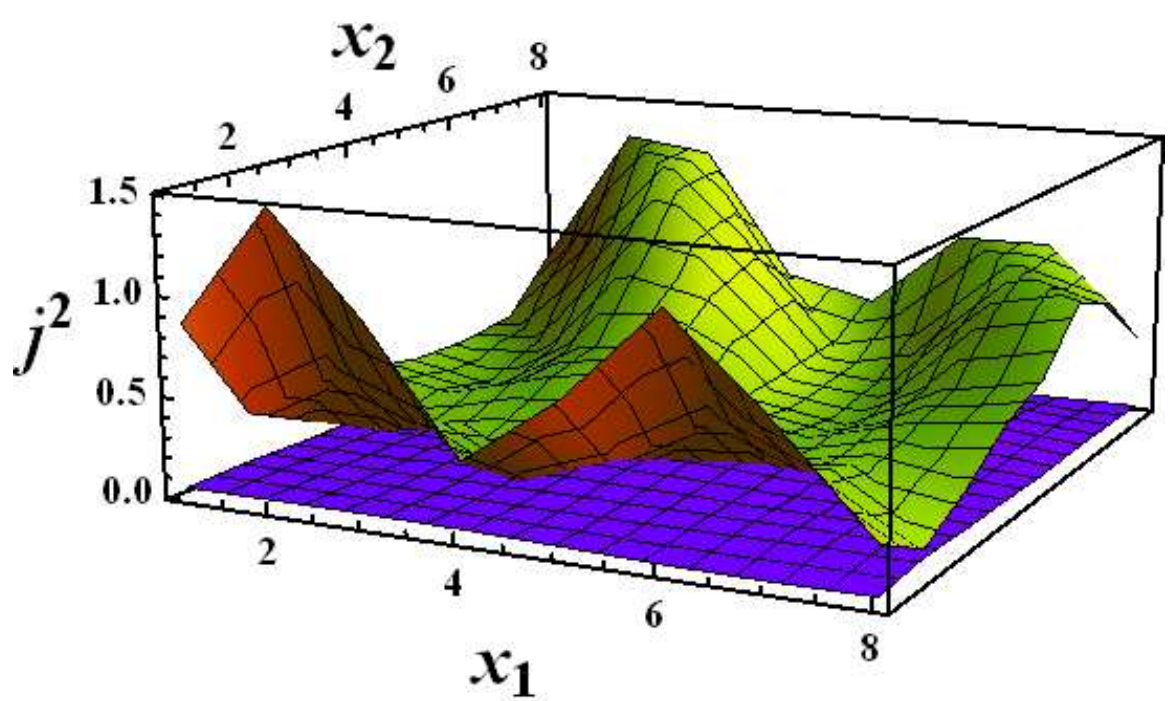

The squared components of the electric current in a 12-plane. The upper sheet represents the spatial distribution of the longitudinal current, the lower sheet corresponds to the transverse current.

$<j_{i}^{2}>_{I R}=<j_{i}^{2}(H, T)>-<j_{i}^{2}(0,0)>, \quad j_{i}=\bar{\psi} \gamma_{i} \psi$ 


\section{$\underline{\text { Conclusions }}$}

- 1. We obsreve signatures of the Chiral Magnetic Effect, but the physics may differ from the model of Kharzeev, McLerran and Warringa (arXiv:0907.0494, Phys.Rev.D79: 106003,2009)

- 2. We observe that in the confinement phase the external magnetic field induces nonzero electric conductivity along the direction of the magnetic field, transforming the system from an insulator into an anisotropic conductor. In the deconfinement phase the conductivity does not exhibit any sizable dependence on the magnetic field (arXiv:1003.2180).

- 3. The conductivity is weaker for heavy quarks, thus it is interesting to measure experimentally the charge asymmetry for $\mathrm{S}$ and $\mathrm{C}$ quarks. 


\section{Conclusions}

- 4. We observe that the chiral condensate is proportional to the strength of the magnetic field, the coefficient of the proportionality agrees with Chiral Perturbation Theory. Microscopic mechanism for the chiral enhancement is the localization of fermion modes in the vacuum (arXiv:0812.1740, Phys.Lett. B 682:484-489,2010 ).

- 5. The calculated vacuum magnetization is in a qualitative agreement with model calculations (arXiv:0906.0488, Nucl.Phys. B 826 (2010) 313).

- 6. We observe very large correlation between electric dipole moment of quark and chirality (arXiv:0909.2350 Phys.Rev.D81:036007,2010). 


\section{Systematic errors}

- SU(2) gluodynamics instead of QCD

- Moderate lattice volumes

- Not large number of gauge field configurations

- In some cases we calculate the overlap propagator using summation over eigenfunctions:

$$
\left\langle\bar{\Psi} \Sigma_{\alpha \beta} \Psi\right\rangle=2 m\left\langle\sum_{\lambda_{k}>0} \frac{\psi_{k}^{\dagger}(x) \Sigma_{\alpha \beta} \psi_{k}(x)}{\lambda_{k}^{2}+m^{2}}\right\rangle
$$

